\title{
ON THE TIME DISCRETIZATION OF STOCHASTIC OPTIMAL CONTROL PROBLEMS: THE DYNAMIC PROGRAMMING APPROACH ${ }^{*, * *, * * *}$
}

\author{
J. Frédéric Bonnans ${ }^{1}$, Justina Gianatti ${ }^{1,2}$ and Francisco J. Silva ${ }^{3}$
}

\begin{abstract}
In this work we consider the time discretization of stochastic optimal control problems. Under general assumptions on the data, we prove the convergence of the value functions associated with the discrete time problems to the value function of the original problem. Moreover, we prove that any sequence of optimal solutions of discrete problems is minimizing for the continuous one. As a consequence of the Dynamic Programming Principle for the discrete problems, the minimizing sequence can be taken in discrete time feedback form.
\end{abstract}

1991 Mathematics Subject Classification. Primary: 93E20, 49L20; Secondary: 90C15, 93C55.

The dates will be set by the publisher.

\section{INTRODUCTION}

Stochastic optimal control problems in continuous time have been extensively studied during the last decades. This important area of research has a wide range of applications, such as in economy, mathematical finance and engineering. Usually, there are two approaches to deal with these problems. The first one is related to the Bellman's Dynamic Programming Principle (DPP), which allows to characterize the value function as the unique viscosity solution of the associated Hamilton-Jacobi-Bellman (HJB) equation [21 23]. The second one is the variational approach, which deals with extensions of the Pontryagin maximum principle 26 to the stochastic framework. For a detailed account of the theory and historical remarks we refer the reader to the classical monographs $14,19,30]$.

Almost independently, another active field of research in the last decades has been the optimal control of discrete time processes and general state space. In that framework, controls at time $k$ (also called policies) are probability measures on the actions space, which depend on the history of states at time $k$ and the chosen

Keywords and phrases: Stochastic Control, Discrete Time Systems, Dynamic Programming Principle, Value Function, Feedback Control

* The first and second author thank the Laboratoire de Finance des Marchs de l'Energie for its support.

** The first and third authors thank the support from project iCODE : "Large-scale systems and Smart grids: distributed decision making" and from the Gaspar Monge Program for Optimization and Operation Research (PGMO).

*** The second author was also supported by Centro Internacional Franco Argentino de Ciencias de la Información y de Sistemas (CIFASIS-CONICET-UNR)

1 Centre de Mathématiques Appliquées, Ecole Polytechnique, INRIA-Saclay, CNRS, Université Paris-Saclay, 91128 Palaiseau, France.

2 CIFASIS - Centro Internacional Franco Argentino de Ciencias de la Información y de Sistemas - CONICET - UNR, S2000EZP Rosario, Argentina

3 Institut de recherche XLIM-DMI, UMR-CNRS 7252, Faculté des sciences et techniques, Université de Limoges, 87060 Limoges, France 
actions up to time $k-1$. Given a control, an action is chosen according to the probability measure associated with this control and this fixes the transition probability function between the states at time $k$ and $k+1$. The literature on this subject is too extensive and we refer the reader to the classical monographs $3,12,15,27,31$, and the references therein, for a comprehensive presentation and historical remarks. Generally speaking, the assumptions in this theory are rather general and a common theme is the investigation of existence of optimal (or $\varepsilon$-optimal) Markov policies, i.e. the chosen control at time $k$ depends only on the value of the state at time $k$.

In this work, we consider a continuous time stochastic optimal control problem with deterministic coefficients and a finite horizon $T>0$. Given a time grid with diameter $h>0$, we study its natural time discretization. While we consider only uniform grids, our analysis is easily extended for general time grids. The state equation is discretized with the classical stochastic explicit Euler scheme. Since at the continuous level we consider the strong formulation for the state equation (see [30, Chapter 2, Section 4.1]), i.e. the control acts pathwise on the state on a fixed probability space, it is natural to consider at the discrete level a similar formulation. In this case, the controls are assumed to be adapted to the filtration generated by the increments of the Brownian motion on the time grid. In this sense and similarly to the continuous time case (see 30, Chapter 2, Section 4.1 and Section 4.2]) our formulation is more specific than the one described in the previous paragraph, which is more related to the weak formulation of the continuous problem (see [30, Chapter 2, Section 4.2]).

The study of the discrete time case arises from different objectives. For instance, it can be used to prove the existence of optimal controls for the continuous time problem, as the limit of optimal discrete time policies (see e.g. 9] and [20]). Another application is to derive the DPP for the continuous time problem as a consequence of this property in the discrete time case (see e.g. [19] and [25]). We point out that in [17, 19] and [25], given a discrete time control the associated state solves the continuous time stochastic differential equation and so the state is not discrete in time. Finally, discrete time problems appear naturally as the first step in obtaining a numerical approximation of the continuous time problem, the second step being the discretization of the state space (see e.g. 20]) or the resolution by Monte Carlo methods.

The simplicity of our pathwise formulation and the regularity of the coefficients defining the continuous problem, which, as we will see, yields the continuity of the optimal cost as a function of the initial state, allow us to simplify the proof of the DPP for the discrete time problem by arguing as in [5]. Thus, we do not have to deal with delicate measurability issues as in 3. Although we consider controls adapted to the filtrations generated by the increments of the Brownian motion, a consequence of the DPP is the existence of discrete time optimal feedback (or Markov) controls. This important property in the discrete time case is in contrast with the analogous property in the continuous time case, where the existence of an optimal feedback control can be assured only in exceptional cases (see [30, Chapter 5, Section 6] and Remark 3.7). In some sense, this is similar to the existence issues for continuous time Stochastic Differential Equations (SDEs), where the Euler scheme is always well-posed even when the continuous time SDEs does not admit explicit solutions.

We study several properties of the discrete time value functions $V^{h}$, which are analogous to their continuous time counterparts, such as Lipschitz continuity and semiconcavity with respect to the state variable on bounded sets. When extended by linear interpolation to the entire interval $[0, T]$, we prove that $V^{h}$ is Hölder continuous in time, on bounded sets of the space variable. Using an approximation result by Krylov (see 19, Lemma 6, Section 3.2, p.143]), we also prove with a direct approach the local uniform convergence of $V^{h}$ to $V$, the value function of the continuous time problem. Since we work under quite general assumptions, this convergence result is more general than those already proved in 9, where under stronger assumptions weak convergence to a feedback control of the continuous time problems is shown, and [14. Chapter 9]. Probably, the convergence of the value functions can also be proved by using analytical methods based on viscosity solution theory (see for instance 7 and 8 for the deterministic case and 2, 11 and 14, Chapter 9] for the stochastic one), however, our direct approach allows us to prove the important fact that optimal (or $\varepsilon$-optimal) discrete time controls form a minimizing sequence for the continuous time problem. In particular, there always exists a minimizing sequence of discrete time optimal feedback controls. In addition, under some convexity (strong convexity) assumptions, we obtain the weak (strong) convergence of the discrete time optimal controls to a solution of the original 
problem. In this general framework, we have not established error estimates for the discrete value functions. We refer the reader to [17] and [18] where, under additional assumptions, the author tackles this problem.

The paper is organized as follows. In Section 2 we state the continuous time and discrete time problems and our main assumptions. Also, we provide some technical and fundamental estimates, which are proved in the Appendix, relating the continuous time and discrete time states associated with piecewise constant controls. In Section 3 we prove the DPP for the discrete time problem and show the existence of feedback optimal controls. The continuity of the discrete value function plays an important role here and simplifies its proof. Next, in Section 4 we prove several regularity properties of $V^{h}$, which are analogous to those of $V$. Finally, in Section 5 we prove in Theorem 5.2 the local uniform convergence of $V^{h}$ to $V$ and that the sequence of discrete time optimal controls is a minimizing sequence of the continuous problem. Under some convexity assumptions, the convergence of this sequence to an optimal control of the continuous problem is also shown.

\section{Preliminaries}

We begin by defining the problems we are interested in.

\subsection{Continuous time problem}

Let $(\Omega, \mathcal{F}, \mathbb{P})$ be a probability space on which an $m$-dimensional standard Brownian motion $W(\cdot)$ is defined. For every $s \in[0, T]$, we set $\mathbb{F}^{s}=\left\{\mathcal{F}_{t}^{s}\right\}_{t \in[s, T]}$ where for $t \in[s, T], \mathcal{F}_{t}^{s}$ is the completion of $\sigma(W(r)-W(s): s \leq$ $r \leq t$ ) by $\mathbb{P}$-null sets of $\mathcal{F}$.

Let $s \in[0, T]$ and $x \in \mathbb{R}^{n}$, we consider the following controlled SDE:

$$
\left\{\begin{aligned}
\mathrm{d} y_{u}^{s, x}(t) & \left.=f\left(t, y_{u}^{s, x}(t), u(t)\right) \mathrm{d} t+\sigma\left(t, y_{u}^{s, x}(t), u(t)\right) \mathrm{d} W(t), \quad t \in\right] s, T[ \\
y_{u}^{s, x}(s) & =x,
\end{aligned}\right.
$$

where $f:[0, T] \times \mathbb{R}^{n} \times \mathbb{R}^{r} \rightarrow \mathbb{R}^{n}$ and $\sigma:[0, T] \times \mathbb{R}^{n} \times \mathbb{R}^{r} \rightarrow \mathbb{R}^{n \times m}$ are given maps. In the notation above $y_{u}^{s, x} \in \mathbb{R}^{n}$ denotes the state function and $u \in \mathbb{R}^{r}$ the control. We define the cost functional

$$
J^{s, x}(u):=\mathbb{E}\left[\int_{s}^{T} \ell\left(t, y_{u}^{s, x}(t), u(t)\right) \mathrm{d} t+g\left(y_{u}^{s, x}(T)\right)\right]
$$

where $\ell:[0, T] \times \mathbb{R}^{n} \times \mathbb{R}^{r} \rightarrow \mathbb{R}$ and $g: \mathbb{R}^{n} \rightarrow \mathbb{R}$ are given maps. A precise definition of the control space, i.e. the domain of the functional $J^{s, x}$ in (2), and assumptions over the data ensuring that $y_{u}^{s, x}$ is well defined will be given in the next sections.

Let $U_{a d}$ be a non-empty compact subset of $\mathbb{R}^{r}$ and define

$$
\mathcal{U}_{a d}^{s}:=\left\{u \in\left(\mathbb{H}_{\mathbb{F}^{s}}^{2}\right)^{r}: u(t, \omega) \in U_{a d} \text {, for almost all (a.a.) }(t, \omega) \in[s, T] \times \Omega\right\},
$$

where

$$
\mathbb{H}_{\mathbb{F}^{s}}^{2}:=\left\{v \in L^{2}([s, T] \times \Omega): \text { the process }(t, \omega) \in[s, T] \times \Omega \mapsto v(t, \omega) \text { is } \mathbb{F}^{s} \text {-adapted }\right\},
$$

and it is endowed with the $L^{2}([s, T] \times \Omega)$ norm.

Then, for fixed $s \in[0, T]$ and $x \in \mathbb{R}^{n}$ the control problem that we consider is

$$
\left(P_{s, x}\right) \quad \inf J^{s, x}(u) \text { subject to } u \in \mathcal{U}_{a d}^{s} \text {. }
$$

The value function of the continuous problem $V:[0, T] \times \mathbb{R}^{n} \rightarrow \mathbb{R}$ is defined as

$$
V(s, x):=\inf _{u \in \mathcal{U}_{a d}^{s}} J^{s, x}(u) .
$$




\subsection{Discrete time problem}

Let us introduce a discrete time approximation of the above problem. Given $N \in \mathbb{N} \backslash\{0\}$, define $h:=T / N$. Let us set $t_{k}=k h(k=0, \ldots, N)$ and consider the sequence of independent and identically distributed (i.i.d.) $m$-valued random vectors, defined as $\Delta W_{k+1}:=W\left(t_{k+1}\right)-W\left(t_{k}\right)$, for $k=0, \ldots, N-1$ and $\Delta W_{0}:=0$ in $\Omega$. Then, $\mathbb{E}\left(\Delta W_{k}\right)=0$ and $\mathbb{E}\left(\Delta W_{k}^{i_{1}} \Delta W_{k}^{i_{2}}\right)=h \delta_{i_{1} i_{2}}$ (where $\delta_{i_{1} i_{2}}=1$ if $i_{1}=i_{2}$ and $\delta_{i_{1} i_{2}}=0$ otherwise). For each $k=0, \ldots, N$ we consider the filtration $\left\{\mathcal{F}_{j}^{k}\right\}_{j=k}^{N}$, where

$$
\mathcal{F}_{k}^{k}:=\{\emptyset, \Omega\} \quad \text { and } \quad \mathcal{F}_{j}^{k}:=\sigma\left(\Delta W_{k^{\prime}} ; k+1 \leq k^{\prime} \leq j\right) \text { for all } j \in\{k+1, \ldots, N\} .
$$

Let us set $L_{\mathcal{F}_{j}^{k}}^{2}:=L^{2}\left(\Omega, \mathcal{F}_{j}^{k}, \mathbb{P}\right)$. For $k=0, \ldots, N-1$, we consider the admissible control sets

$$
\mathcal{U}_{k}^{h}:=\left\{u=\left(u_{k}, \ldots, u_{N-1}\right) \in \Pi_{j=k}^{N-1} L_{\mathcal{F}_{j}^{k}}^{2}: u_{j}(\omega) \in U_{a d} \quad \mathbb{P} \text {-a.s., } j=k, \ldots, N-1\right\},
$$

where $\Pi_{j=k}^{N-1} L_{\mathcal{F}_{j}^{k}}^{2}$ is endowed with the norm $\|u\|_{\mathcal{U}_{k}^{h}}^{2}:=h \sum_{j=k}^{N-1} \mathbb{E}\left|u_{j}\right|^{2}$.

Given $u \in \mathcal{U}_{k}^{h}, x \in \mathbb{R}^{n}$ and $k=0, \ldots, N-1$, we recursively define the state $y_{j}^{k, x, u}, j=k, \ldots, N$, associated with $u, x$ and $k$ as

$$
\left\{\begin{array}{l}
y_{j+1}^{k, x, u}=y_{j}^{k, x, u}+h f\left(t_{j}, y_{j}^{k, x, u}, u_{j}\right)+\sigma\left(t_{j}, y_{j}^{k, x, u}, u_{j}\right) \Delta W_{j+1}, \quad j=k, \ldots, N-1, \\
y_{k}^{k, x, u}=x .
\end{array}\right.
$$

Note that, under the assumption (H1) below, $y_{j}^{k, x, u} \in L_{\mathcal{F}_{j}^{k}}^{2}$, for all $j=k, \ldots, N$.

Finally, we associate to each $u \in \mathcal{U}_{k}^{h}$ the cost function,

$$
J_{k}^{h}(x, u):=\mathbb{E}\left[h \sum_{j=k}^{N-1} \ell\left(t_{j}, y_{j}^{k, x, u}, u_{j}\right)+g\left(y_{N}^{k, x, u}\right)\right] .
$$

Then, for fixed $k$ and $x$, the discrete time control problem that we will consider is

$$
\left(P_{k, x}^{h}\right) \quad \inf J_{k}^{h}(x, u) \text { subject to } u \in \mathcal{U}_{k}^{h} \text {. }
$$

In this case, the value function $\left\{V_{k}^{h}: \mathbb{R}^{n} \rightarrow \mathbb{R} \mid k=0, \ldots, N\right\}$ is defined over $\mathbb{R}^{n}$ as

$$
V_{N}^{h}(x):=g(x), \quad \text { and } \quad V_{k}^{h}(x):=\inf _{u \in \mathcal{U}_{k}^{h}} J_{k}^{h}(x, u) \text { for } k=0, \ldots, N-1 .
$$

The main result of this work is to prove the convergence of an extension of $V_{(\cdot)}^{h}(\cdot)$ to $[0, T] \times \mathbb{R}^{n}$ to the value function $V$. In the next section we introduce the main assumptions in this work.

\subsection{Assumptions}

We present the hypothesis that we consider in this paper.

(H1) Assumptions on the dynamics:

(a) The maps $\varphi=f, \sigma$ are $\mathcal{B}\left([0, T] \times \mathbb{R}^{n} \times \mathbb{R}^{r}\right)$ measurable.

(b) For almost all $t \in[0, T]$ the map $(y, u) \mapsto \varphi(t, y, u)$ is $C^{1}$ and there exists a constant $L_{1}>0$ such that for almost all $t \in[0, T]$ and for all $y \in \mathbb{R}^{n}$ and $u \in U_{a d}$ we have

$$
\left\{\begin{array}{l}
|\varphi(t, y, u)| \leq L_{1}[|y|+|u|+1] \\
\left|\varphi_{y}(t, y, u)\right|+\left|\varphi_{u}(t, y, u)\right| \leq L_{1}
\end{array}\right.
$$


where $\varphi_{y}(t, y, u):=D_{y} \varphi(t, y, u)$ and $\varphi_{u}(t, y, u):=D_{u} \varphi(t, y, u)$.

(c) There exists an increasing modulus of continuity $\bar{\omega}_{1}:[0,+\infty[\rightarrow[0,+\infty[$ such that for $\varphi=f, \sigma$, and for all $y \in \mathbb{R}^{n}, u \in U_{a d}, s, t \in[0, T]$ we have

$$
|\varphi(s, y, u)-\varphi(t, y, u)| \leq \bar{\omega}_{1}(|s-t|) .
$$

(H2) Assumptions on the cost:

(a) The maps $\ell$ and $g$ are respectively $\mathcal{B}\left([0, T] \times \mathbb{R}^{n} \times \mathbb{R}^{r}\right)$ and $\mathcal{B}\left(\mathbb{R}^{n}\right)$ measurable.

(b) For almost all $t \in[0, T]$ the map $(y, u) \mapsto \ell(t, y, u)$ is $C^{1}$, and there exists $L_{2}>0$ such that for all $y \in \mathbb{R}^{n}$ and $u \in U_{a d}$

$$
\left\{\begin{array}{l}
|\ell(t, y, u)| \leq L_{2}[|y|+|u|+1]^{2}, \\
\left|\ell_{y}(t, y, u)\right|+\left|\ell_{u}(t, y, u)\right| \leq L_{2}[|y|+|u|+1],
\end{array}\right.
$$

where $\ell_{y}(t, y, u):=D_{y} \ell(t, y, u)$ and $\ell_{u}(t, y, u):=D_{u} \ell(t, y, u)$.

(c) There exists an increasing modulus of continuity $\bar{\omega}_{2}:\left[0,+\infty\left[\rightarrow\left[0,+\infty\left[\right.\right.\right.\right.$ such that for for all $y \in \mathbb{R}^{n}$, $u \in U_{a d}$ and $s, t \in[0, T]$ we have

$$
|\ell(s, y, u)-\ell(t, y, u)| \leq \bar{\omega}_{2}(|s-t|) .
$$

(d) The map $y \mapsto g(y)$ is $C^{1}$ and there exists $L_{2}>0$ such that for all $y \in \mathbb{R}^{n}$,

$$
\left\{\begin{array}{l}
|g(y)| \leq L_{2}[|y|+1]^{2} \\
|\nabla g(y)| \leq L_{2}[|y|+1]
\end{array}\right.
$$

In order to keep the notation as simple as possible, we define $L:=\max \left\{L_{1}, L_{2}\right\}$ and $\bar{\omega}:=\max \left\{\bar{\omega}_{1}, \bar{\omega}_{2}\right\}$.

Remark 2.1. Under assumption (H1), for any $u \in \mathcal{U}_{a d}^{s}$ the state equation (1) admits a unique strong solution, see the proof of [24, Proposition 2.1]. For the sake of completeness, we recall the following particular instance of [24, Proposition 2.1] which is valid under our assumption (H1).

Proposition 2.1 Assume that (H1) holds true. Then for any $u \in \mathcal{U}_{a d}^{0}$ the continuous times state equation (1) admits a unique strong solution $y \in L^{2}\left(\Omega ; C\left([0, T] ; \mathbb{R}^{n}\right)\right)$, and the following estimate holds:

$$
\mathbb{E}\left[\sup _{s \in[0, t]}|y(s)|^{2}\right] \leq C \mathbb{E}\left[|x|^{2}+\left(\int_{0}^{t}|f(s, 0, u(s))| \mathrm{d} s\right)^{2}+\int_{0}^{t}|\sigma(s, 0, u(s))|^{2} \mathrm{~d} s\right] .
$$

Moreover, for any $\gamma \geq 1$, there exists $C_{\gamma}>0$ such that

$$
\mathbb{E}\left[\sup _{s \in[0, t]}\left|y_{u}^{0, x}(s)-y_{u}^{0, \bar{x}}(s)\right|^{\gamma}\right] \leq C_{\gamma}|x-\bar{x}|^{\gamma}
$$

Under assumptions (H1) and (H2) we can prove the main result presented in Section 5 (Theorem [5.2), which gives the convergence of the discrete value function to the continuous one. The contribution of this work in the context of the existing literature is that our assumptions are rather general, see for instance [1] where the coefficient are bounded. Moreover, using the DPP, proved in Section 3, we obtain the existence of feedback discrete optimal controls which will be shown to form a minimizing sequence for the continuous problem.

We end this section by providing some technical results which will be needed in the next sections. 


\subsection{Estimates on the states of the discrete and continuous formulations}

We begin this section by providing some estimates for the discrete state and then for the difference between two discrete states with different initial state. Finally, we also prove some estimates for the difference between the discrete and continuous time states associated with the same discrete time control, which is extended to a piecewise constant control at each interval $\left[t_{k}, t_{k+1}[\right.$. The proofs of these results will be given in the Appendix.

In order to keep the notation as simple as possible, we assume that the initial time is $k=0$ and the initial state $x$ is fixed, and we omit these indexes in the state.

We start by providing an estimate of the norm of the state in terms of the control and the initial condition. For a fixed $h$, let $u^{h}=\left(u_{k}\right)_{k=0}^{N-1} \in \mathcal{U}_{0}^{h}$ be a given discrete control and define the associated discrete state $y^{h}=\left(y_{k}\right)_{k=0}^{N}$ as the solution of (8).

Let us underline that all the constants involved in the following results are independent of $h$.

Lemma 2.2. Assume that (H1) holds. Then, there exists $C>0$, such that

$$
\mathbb{E}\left[\max _{k=0, \ldots, N}\left|y_{k}\right|^{2}\right] \leq C\left[|x|^{2}+\left\|u^{h}\right\|_{\mathcal{U}_{0}^{h}}^{2}+1\right] .
$$

Proof. See the Appendix.

Lemma 2.3. Let (H1) holds. Then, for every $p \geq 2$, there exists $C_{p}>0$, such that for all $x, y \in \mathbb{R}^{n}$, we have

$$
\mathbb{E}\left[\max _{k=0, \ldots, N}\left|y_{k}^{x}-y_{k}^{y}\right|^{p}\right] \leq C_{p}|x-y|^{p}
$$

where $\left(y_{k}^{x}\right)_{k=0}^{N}$ and $\left(y_{k}^{y}\right)_{k=0}^{N}$ are the solutions of (8) associated with the control $u^{h}$ and the initial states $x$ and $y$, respectively.

Proof. See the Appendix.

Let $u_{c}^{h} \in \mathbb{H}_{\mathbb{F}}^{2}$ be defined as $u_{c}^{h}(t):=u_{k}$ for all $t \in\left[t_{k}, t_{k+1}\right)$ and $y$ be the associated continuous state, i.e. the unique solution of

$$
\left\{\begin{aligned}
\mathrm{d} y(t) & \left.=f\left(t, y(t), u_{c}^{h}(t)\right) \mathrm{d} t+\sigma\left(t, y(t), u_{c}^{h}(t)\right) \mathrm{d} W(t), \quad t \in\right] 0, T[ \\
y(0) & =x
\end{aligned}\right.
$$

The existence and uniqueness of solution associated with $u_{c}^{h}$ is guaranteed by Remark 2.1, Now, we are going to analyse the relationship between $y$, the solution of (20) associated with $u_{c}^{h}$, and $y^{h}$, the solution of (8) associated with $u^{h}$. Note that $\left\|u_{c}^{h}\right\|_{\mathbb{H}_{\mathbb{F}^{0}}^{2}}=\left\|u^{h}\right\|_{\mathcal{U}_{0}^{h}}$.

Lemma 2.4. Assume that (H1) holds true. Then, there exists $C>0$ such that for all $k=0, \ldots, N-1$,

$$
\mathbb{E}\left[\sup _{t_{k} \leq t<t_{k+1}}\left|y(t)-y_{k}\right|^{2}\right] \leq C h\left[|x|^{2}+\left\|u^{h}\right\|_{\mathcal{U}_{0}^{h}}^{2}+1\right]+C \bar{\omega}^{2}(h) .
$$

Proof. See the Appendix. 


\section{Discrete Time Dynamic Programming Principle}

Throughout this section we consider $h=T / N$ fixed. Our goal in this section is to prove the DPP for problem $\left(P_{k, x}^{h}\right)$ parameterized by the initial discrete time $k$ and the initial state $x$. Besides its own importance, the DPP implies the existence of discrete time optimal feedback controls, and it is also useful to prove the convergence of the discrete value functions to the continuous one, as we will see in Section 5 .

Our aim is to prove the following DPP for $\left\{V_{k}^{h}: k=0, \ldots, N\right\}$

$$
V_{k}^{h}(x)=\inf _{u \in U_{a d}}\left\{h \ell\left(t_{k}, x, u\right)+\mathbb{E}\left[V_{k+1}^{h}\left(x+h f\left(t_{k}, x, u\right)+\sigma\left(t_{k}, x, u\right) \Delta W_{k+1}\right)\right]\right\},
$$

for all $k=0, \ldots, N-1$, where we recall that $\left\{V_{k}^{h}: k=0, \ldots, N\right\}$ was defined in 10 . Note that (H2), the compactness of $U_{a d}$ and Lemma 2.2 imply that $V_{k}^{h}(x) \in \mathbb{R}$ for all $k=0, \ldots, N$ and $x \in \mathbb{R}^{n}$.

We will need the following result which proves that the discrete value function is Lipschitz continuous with respect to the state variable, on bounded sets.

Lemma 3.1. Assume that (H1) and (H2) hold. Then, there exists a constant $C>0$ (independent of $h$ ), such that for all $x, y \in \mathbb{R}^{n}$, and for all $u \in \mathcal{U}_{k}^{h}$, we have

$$
\left|J_{k}^{h}(x, u)-J_{k}^{h}(y, u)\right| \leq C[|x|+|y|+1]|x-y|, \quad \forall k=0, \ldots, N-1 .
$$

As a consequence,

$$
\left|V_{k}^{h}(x)-V_{k}^{h}(y)\right| \leq C[|x|+|y|+1]|x-y| \quad \forall k=0, \ldots, N .
$$

Proof. By notational convenience, we omit the indexes $k$ and $u$ in the states. We have for fixed $k$ and $u \in \mathcal{U}_{k}^{h}$

$$
\left|J_{k}^{h}(x, u)-J_{k}^{h}(y, u)\right| \leq \mathbb{E}\left[h \sum_{j=k}^{N-1}\left|\ell\left(t_{j}, y_{j}^{x}, u_{j}\right)-\ell\left(t_{j}, y_{j}^{y}, u_{j}\right)\right|+\left|g\left(y_{N}^{x}\right)-g\left(y_{N}^{y}\right)\right|\right] .
$$

By (H2) we have for all $k \leq j \leq N-1$,

$$
\begin{aligned}
\mathbb{E}\left[\left|\ell\left(t_{j}, y_{j}^{x}, u_{j}\right)-\ell\left(t_{j}, y_{j}^{y}, u_{j}\right)\right|\right] & \leq \mathbb{E}\left[\int_{0}^{1}\left|\ell_{y}\left(t_{j}, y_{j}^{x}+s\left(y_{j}^{y}-y_{j}^{x}\right), u_{j}\right)\left(y_{j}^{y}-y_{j}^{x}\right)\right| \mathrm{d} s\right] \\
& \leq \mathbb{E}\left[\int_{0}^{1}\left[L\left(1+\left|y_{j}^{x}\right|+s\left|y_{j}^{y}-y_{j}^{x}\right|+\left|u_{j}\right|\right)\left|y_{j}^{y}-y_{j}^{x}\right|\right] \mathrm{d} s\right] \\
& \leq L \mathbb{E}\left[\left|y_{j}^{y}-y_{j}^{x}\right|+\left|y_{j}^{x}\right|\left|y_{j}^{y}-y_{j}^{x}\right|+\left|y_{j}^{y}-y_{j}^{x}\right|^{2}+\left|u_{j}\right|\left|y_{j}^{y}-y_{j}^{x}\right|\right] .
\end{aligned}
$$

Since the set $U_{a d}$ is compact, it is bounded by a positive constant $M_{U}$, and so by the Cauchy-Schwarz inequality, Lemma 2.2 and Lemma 2.3 there exists $C_{0}>0$ such that

$$
\begin{aligned}
& \mathbb{E}\left[\left|y_{j}^{y}-y_{j}^{x}\right|\right] \leq\left(\mathbb{E}\left[\left|y_{j}^{y}-y_{j}^{x}\right|^{2}\right]\right)^{\frac{1}{2}} \leq C_{0}|x-y|, \\
& \mathbb{E}\left[\left|y_{j}^{x}\right|\left|y_{j}^{y}-y_{j}^{x}\right|\right] \leq\left(\mathbb{E}\left[\left|y_{j}^{x}\right|^{2}\right]\right)^{\frac{1}{2}}\left(\mathbb{E}\left[\left|y_{j}^{y}-y_{j}^{x}\right|^{2}\right]\right)^{\frac{1}{2}} \leq C_{0}\left[|x|^{2}+T M_{U}^{2}+1 \mid\right]^{\frac{1}{2}}|x-y|, \\
& \mathbb{E}\left[\left|u_{j}\right|\left|y_{j}^{y}-y_{j}^{x}\right|\right] \leq\left(\mathbb{E}\left[\left|u_{j}\right|^{2}\right]\right)^{\frac{1}{2}}\left(\mathbb{E}\left[\left|y_{j}^{y}-y_{j}^{x}\right|^{2}\right]\right)^{\frac{1}{2}} \leq C_{0} M_{U}|x-y|, \\
& \mathbb{E}\left[\left|y_{j}^{y}-y_{j}^{x}\right|^{2}\right] \leq C_{0}|x-y|^{2} \leq C_{0}[|x|+|y|]|x-y| .
\end{aligned}
$$

We conclude that there exists $C_{1}>0$ such that,

$$
\mathbb{E}\left[\left|\ell\left(t_{j}, y_{j}^{x}, u_{j}\right)-\ell\left(t_{j}, y_{j}^{y}, u_{j}\right)\right|\right] \leq C_{1}[|x|+|y|+1]|x-y| .
$$


Analogously we can deduce that

$$
\mathbb{E}\left[\left|g\left(y_{N}^{x}\right)-g\left(y_{N}^{y}\right)\right|\right] \leq C_{1}[|x|+|y|+1]|x-y| .
$$

Since $C_{1}$ in 28$)$ does not depend on $j$, we conclude that

$$
\left|J_{k}^{h}(x, u)-J_{k}^{h}(y, u)\right| \leq(T+1) C_{1}[|x|+|y|+1]|x-y|,
$$

and 23 follows.

Since the set $\mathcal{U}_{k}^{h}$ is bounded, and the constant $C_{1}$ obtained is independent of $u \in \mathcal{U}_{k}^{h}$, relation 24 easily follows from the inequality

$$
\left|V_{k}^{h}(x)-V_{k}^{h}(y)\right| \leq \sup _{u \in \mathcal{U}_{k}^{h}}\left|J_{k}^{h}(x, u)-J_{k}^{h}(y, u)\right| .
$$

In order to prove the DPP $(22)$, for all $x \in \mathbb{R}^{n}$ we define

$$
\begin{aligned}
& S_{k}^{h}(x):=\inf _{u \in U_{a d}}\left\{h \ell\left(t_{k}, x, u\right)+\mathbb{E}\left[V_{k+1}^{h}\left(y_{k+1}^{k, x, u}\right)\right]\right\}, \quad \forall k=0, \ldots, N-1, \\
& S_{N}^{h}(x):=g(x),
\end{aligned}
$$

where,

$$
y_{k+1}^{k, x, u}=x+h f\left(t_{k}, x, u\right)+\sigma\left(t_{k}, x, u\right) \Delta W_{k+1} .
$$

Lemma 3.2. For all $x \in \mathbb{R}^{n}$, and for all $k=0, \ldots, N$, the map $u \in U_{a d} \mapsto \mathbb{E}\left[V_{k+1}^{h}\left(y_{k+1}^{k, x, u}\right)\right]$ is Lipschitz continuous. As a consequence, $S_{k}^{h}(x) \in \mathbb{R}$ for all $x \in \mathbb{R}^{n}$, and there exists $u_{k, x} \in U_{\text {ad }}$ where the minimum in the first relation in $(32)$ is attained.

Proof. Let $u, v \in U_{a d}$, then by (H1) and the Itô isometry,

$$
\begin{aligned}
\mathbb{E}\left[\left|y_{k+1}^{k, x, u}-y_{k+1}^{k, x, v}\right|^{2}\right] & \leq \mathbb{E}\left[\left|h\left(f\left(t_{k}, x, u\right)-f\left(t_{k}, x, v\right)\right)+\left(\sigma\left(t_{k}, x, u\right)-\sigma\left(t_{k}, x, v\right)\right) \Delta W_{k+1}\right|^{2}\right] \\
& \leq 2 h^{2} \mathbb{E}\left[\left|f\left(t_{k}, x, u\right)-f\left(t_{k}, x, v\right)\right|^{2}\right]+2 h \mathbb{E}\left[\left|\sigma\left(t_{k}, x, u\right)-\sigma\left(t_{k}, x, v\right)\right|^{2}\right] \\
& \leq 2 h^{2} L^{2}|u-v|^{2}+2 h L^{2}|u-v|^{2} .
\end{aligned}
$$

Thus, by Lemma 2.2. Lemma 3.1 and the Cauchy-Schwarz inequality, there exist $C_{0}>0$ and $C>0$ such that,

$$
\begin{aligned}
\left|\mathbb{E}\left[V_{k+1}^{h}\left(y_{k+1}^{k, x, u}\right)-V_{k+1}^{h}\left(y_{k+1}^{k, x, v}\right)\right]\right| & \leq \mathbb{E}\left[\left|C_{0}\left[\left|y_{k+1}^{k, x, u}\right|+\left|y_{k+1}^{k, x, v}\right|+1\right]\right| y_{k+1}^{k, x, u}-y_{k+1}^{k, x, v} \mid\right] \\
& \leq\left(\mathbb{E}\left[\left|C_{0}\left[\left|y_{k+1}^{k, x, u}\right|+\left|y_{k+1}^{k, x, v}\right|+1\right]\right|^{2}\right]\right)^{\frac{1}{2}}\left(\mathbb{E}\left[\left|y_{k+1}^{k, x, u}-y_{k+1}^{k, x, v}\right|^{2}\right]\right)^{\frac{1}{2}} \\
& \leq C[|x|+1]|u-v| .
\end{aligned}
$$

By (35), we conclude that $U_{a d} \ni u \mapsto \mathbb{E}\left[V_{k+1}^{h}\left(y_{k+1}^{k, x, u}\right)\right]$ is locally Lipschitz continuous, and, hence, using (H2) and that $U_{a d}$ is compact, we get that $S_{k}^{h}(x)$ is finite and the minimum in 32 is attained.

Lemma 3.3. Under assumptions (H1) and (H2), there exists $C>0$ (independent of $h$ ), such that for all $x, y \in \mathbb{R}^{n}$, and $k=0, \ldots, N-1$,

$$
\left|S_{k}^{h}(x)-S_{k}^{h}(y)\right| \leq C[|x|+|y|+1]|x-y|
$$


Proof. By the definition of $S_{k}^{h}$ and Lemma 3.1, there exists $C_{0}>0$ such that

$$
\begin{aligned}
\left|S_{k}^{h}(x)-S_{k}^{h}(y)\right| & \leq \sup _{u \in U_{a d}}\left\{h\left|\ell\left(t_{k}, x, u\right)-\ell\left(t_{k}, y, u\right)\right|+\mathbb{E}\left[\left|V_{k+1}^{h}\left(y_{k+1}^{k, x, u}\right)-V_{k+1}^{h}\left(y_{k+1}^{k, y, u}\right)\right|\right]\right\} \\
& \leq h C_{0}[|x|+|y|+1]|x-y|+\mathbb{E}\left[C_{0}\left[\left|y_{k+1}^{k, x, u}\right|+\left|y_{k+1}^{k, y, u}\right|+1\right]\left|y_{k+1}^{k, x, u}-y_{k+1}^{k, y, u}\right|\right] .
\end{aligned}
$$

By the Cauchy-Schwarz inequality, we obtain

$$
\mathbb{E}\left[\left[\left|y_{k+1}^{k, x, u}\right|+\left|y_{k+1}^{k, y, u}\right|+1\right]\left|y_{k+1}^{k, x, u}-y_{k+1}^{k, y, u}\right|\right] \leq\left(\mathbb{E}\left[\left[\left|y_{k+1}^{k, x, u}\right|+\left|y_{k+1}^{k, y, u}\right|+1\right]^{2}\right]\right)^{\frac{1}{2}}\left(\mathbb{E}\left[\left|y_{k+1}^{k, x, u}-y_{k+1}^{k, y, u}\right|^{2}\right]\right)^{\frac{1}{2}}
$$

By Lemma 2.2 and Lemma 2.3 we conclude that there exists $C_{1}>0$, independent of $u$, because $U_{a d}$ is bounded, such that

$$
\left(\mathbb{E}\left[\left[\left|y_{k+1}^{k, x, u}\right|+\left|y_{k+1}^{k, y, u}\right|+1\right]^{2}\right]\right)^{\frac{1}{2}}\left(\mathbb{E}\left[\left|y_{k+1}^{k, x, u}-y_{k+1}^{k, y, u}\right|^{2}\right]\right)^{\frac{1}{2}} \leq C_{1}[|x|+|y|+1]|x-y| .
$$

Combining (37) and (39), we deduce that (36) holds true.

Now we prove the DPP. Since $V_{k}^{h}$ is continuous, we can directly prove the result following the arguments in [5] without needing to embed our problem in the general framework of 3 .

Theorem 3.4 (DPP). Under assumptions (H1) and (H2), for all $x \in \mathbb{R}^{n}$ we have

$$
V_{k}^{h}(x)=S_{k}^{h}(x) \quad \forall k=0, \ldots, N
$$

Proof. We start by proving that $V_{k}^{h}(x) \geq S_{k}^{h}(x)$. Let $u=\left(u_{k}, \ldots, u_{N-1}\right)$ be any element of $\mathcal{U}_{k}^{h}$. By the definition of the set $\mathcal{U}_{k}^{h}$, we can write $u_{j}$ for $j \in\{k+1, \ldots, N-1\}$, as a measurable function of the increments of the Brownian motion, i.e. $u_{j}\left(\Delta W_{k+1}, \ldots, \Delta W_{j}\right)$. For fixed $\Delta \omega_{k+1} \in \mathbb{R}^{m}$ and $j=k+1, \ldots, N-1$, we define the maps

$$
\hat{u}_{j}\left(\Delta \omega_{k+1}\right): \omega \in \Omega \mapsto u_{j}\left(\Delta \omega_{k+1}, \Delta W_{k+2}(\omega), \ldots, \Delta W_{j}(\omega)\right) .
$$

Setting $\hat{u}\left(\Delta \omega_{k+1}\right)=\left(\hat{u}_{k+1}\left(\Delta \omega_{k+1}\right), \ldots, \hat{u}_{N-1}\left(\Delta \omega_{k+1}\right)\right)$, we obtain $\hat{u}\left(\Delta \omega_{k+1}\right) \in \mathcal{U}_{k+1}^{h}$. Then, we can also define,

$$
\hat{y}_{k+1}\left(\Delta \omega_{k+1}\right):=x+h f\left(t_{k}, x, u_{k}\right)+\sigma\left(t_{k}, x, u_{k}\right) \Delta \omega_{k+1},
$$

which is deterministic, and for $j=k+2, \ldots, N$,

$$
\hat{y}_{j}\left(\Delta \omega_{k+1}\right): \omega \in \Omega \mapsto y_{j}^{k+1, \hat{y}_{k+1}\left(\Delta \omega_{k+1}\right), \hat{u}\left(\Delta \omega_{k+1}\right)(\omega)}(\omega) .
$$

Then, by the independence of the increments of a Brownian motion, we have

$$
\begin{aligned}
\mathbb{E}\left[h \sum_{j=k+1}^{N-1} \ell\left(t_{j}, y_{j}^{k, x, u}, u_{j}\right)+g\left(y_{N}^{k, x, u}\right)\right]= \\
\int_{\mathbb{R}^{m}} \mathbb{E}\left[h \sum_{j=k+1}^{N-1} \ell\left(t_{j}, \hat{y}_{j}\left(\Delta \omega_{k+1}\right), \hat{u}_{j}\left(\Delta \omega_{k+1}\right)\right)+g\left(\hat{y}_{N}\left(\Delta \omega_{k+1}\right)\right)\right] \mathrm{d} \mathbb{P}_{\Delta W_{k+1}}\left(\Delta \omega_{k+1}\right),
\end{aligned}
$$


where $\mathbb{P}_{\Delta W_{k+1}}$ is the measure induced from $\mathbb{P}$ by $\Delta W_{k+1}$ on $\mathbb{R}^{m}$ (see 1 , Section 4.13]). Thus,

$$
\begin{aligned}
\mathbb{E}\left[h \sum_{j=k+1}^{N-1} \ell\left(t_{j}, y_{j}^{k, x, u}, u_{j}\right)+g\left(y_{N}^{k, x, u}\right)\right] \\
=\int_{\mathbb{R}^{m}}\left(h \ell\left(t_{k+1}, \hat{y}_{k+1}\left(\Delta \omega_{k+1}\right), \hat{u}_{k+1}\left(\Delta \omega_{k+1}\right)\right)\right. \\
\left.\quad \quad+\mathbb{E}\left[h \sum_{j=k+2}^{N-1} \ell\left(t_{j}, \hat{y}_{j}\left(\Delta \omega_{k+1}\right), \hat{u}_{j}\left(\Delta \omega_{k+1}\right)\right)+g\left(\hat{y}_{N}\left(\Delta \omega_{k+1}\right)\right)\right]\right) \mathrm{d} \mathbb{P}_{\Delta W_{k+1}}\left(\Delta \omega_{k+1}\right) \\
\geq \int_{\mathbb{R}^{m}} V_{k+1}^{h}\left(\hat{y}_{k+1}\left(\Delta \omega_{k+1}\right)\right) \mathrm{d} \mathbb{P}_{\Delta W_{k+1}}\left(\Delta \omega_{k+1}\right) \\
=\mathbb{E}\left[V_{k+1}^{h}\left(x+h f\left(t_{k}, x, u_{k}\right)+\sigma\left(t_{k}, x, u_{k}\right) \Delta W_{k+1}\right)\right] .
\end{aligned}
$$

Since $u_{k} \in L_{\mathcal{F}_{k}^{k}}^{2}$ and $\mathcal{F}_{k}^{k}=\{\emptyset, \Omega\}$, for all $u=\left(u_{k}, \ldots, u_{N-1}\right) \in \mathcal{U}_{k}^{h}$ we have

$$
\begin{aligned}
\ell\left(t_{k}, x, u_{k}\right)+ & \mathbb{E}\left[h \sum_{j=k+1}^{N-1} \ell\left(t_{j}, y_{j}^{k, x, u}, u_{j}\right)+g\left(y_{N}^{k, x, u}\right)\right] \\
& \geq \ell\left(t_{k}, x, u_{k}\right)+\mathbb{E}\left[V_{k+1}^{h}\left(x+h f\left(t_{k}, x, u_{k}\right)+\sigma\left(t_{k}, x, u_{k}\right) \Delta W_{k+1}\right)\right] \\
& \geq S_{k}^{h}(x) .
\end{aligned}
$$

Minimizing w.r.t. $u \in \mathcal{U}_{k}^{h}$ in the l.h.s. we deduce

$$
V_{k}^{h}(x) \geq S_{k}^{h}(x) .
$$

We next prove the converse inequality by an induction argument. It is clear by the definitions that

$$
V_{N}^{h}(x)=S_{N}^{h}(x) \text { and } V_{N-1}^{h}(x)=S_{N-1}^{h}(x), \quad \forall x \in \mathbb{R}^{n} .
$$

Now, let $\varepsilon$ be a positive number and for each $x \in \mathbb{R}^{n}$ let $\delta_{x}>0$ be such that $C[|x|+|y|+1]|x-y|<\frac{\varepsilon}{2}$ for all $y:|x-y|<\delta_{x}$, where $C$ is the maximum of the constants given in Lemma 3.1 and Lemma 3.3, Then, for all $k=0, \ldots, N$ and $u \in \mathcal{U}_{k}^{h}$,

$$
\max \left\{\left|J_{k}^{h}(x, u)-J_{k}^{h}(y, u)\right|,\left|V_{k}^{h}(x)-V_{k}^{h}(y)\right|,\left|S_{k}^{h}(x)-S_{k}^{h}(y)\right|\right\}<\frac{\varepsilon}{2}, \quad \forall y:|x-y|<\delta_{x} .
$$

Since $\mathbb{R}^{n}$ is a Lindelöf space, i.e. every open cover has a countable subcover, there exists a sequence $\left(\xi_{i}\right)_{i \in \mathbb{N}} \subset \mathbb{R}^{n}$ such that $\mathbb{R}^{n}=\bigcup_{i=1}^{\infty} B\left(\xi_{i}, \delta_{\xi_{i}}\right)$. In order to obtain a disjoint union, we can define

$$
\hat{B}_{1}:=B\left(\xi_{1}, \delta_{\xi_{1}}\right), \quad \text { and } \quad \hat{B}_{i}:=B\left(\xi_{i}, \delta_{\xi_{i}}\right) \backslash\left(\cup_{j=1}^{i-1} \hat{B}_{j}\right), \quad \forall i>1 .
$$

Let $k<N-1$, and assume that $V_{n}^{h} \equiv S_{n}^{h}$, for all $n=k+1, \ldots, N$. Since $U_{a d}$ is compact, by Lemma 3.2, for $j=k, \ldots, N-1$, there exists $u_{j}^{i} \in U_{a d}$ such that

$$
h \ell\left(t_{j}, \xi_{i}, u_{j}^{i}\right)+\mathbb{E}\left[V_{j+1}^{h}\left(\xi_{i}+h f\left(t_{j}, \xi_{i}, u_{j}^{i}\right)+\sigma\left(t_{j}, \xi_{i}, u_{j}^{i}\right) \Delta W_{j+1}\right)\right]=S_{j}^{h}\left(\xi_{i}\right) .
$$

We define the measurable function $u_{j}(x)=\sum_{i=1}^{\infty} u_{j}^{i} \chi_{\hat{B}_{i}}(x)$. Let $x \in \mathbb{R}^{n}$ and $i$ such that $x \in \hat{B}_{i}$ (see (50)). Then,

$$
\begin{aligned}
h \ell\left(t_{j}, x, u_{j}(x)\right) & +\mathbb{E}\left[V_{j+1}^{h}\left(x+h f\left(t_{j}, x, u_{j}(x)\right)+\sigma\left(t_{j}, x, u_{j}(x)\right) \Delta W_{j+1}\right)\right] \\
= & h \ell\left(t_{j}, x, u_{j}^{i}\right)+\mathbb{E}\left[V_{j+1}^{h}\left(x+h f\left(t_{j}, x, u_{j}^{i}\right)+\sigma\left(t_{j}, x, u_{j}^{i}\right) \Delta W_{j+1}\right)\right] \\
\leq & \frac{\varepsilon}{2}+h \ell\left(t_{j}, \xi_{i}, u_{j}^{i}\right)+\mathbb{E}\left[V_{j+1}^{h}\left(\xi_{i}+h f\left(t_{j}, \xi_{i}, u_{j}^{i}\right)+\sigma\left(t_{j}, \xi_{i}, u_{j}^{i}\right) \Delta W_{j+1}\right)\right] \\
\leq & \frac{\varepsilon}{2}+S_{j}^{h}\left(\xi_{i}\right) \\
\leq & S_{j}^{h}(x)+\varepsilon .
\end{aligned}
$$


Now, we fix $x \in \mathbb{R}^{n}$ and take $\bar{u}_{k}=u_{k}(x) \in U_{a d}$. We define inductively $u_{j}: \Omega \rightarrow \mathbb{R}^{r}$, for all $k<j \leq N-1$, as

$$
\bar{u}_{j+1}(\omega):=u_{j+1}\left(y_{j+1}^{k, x,\left(\bar{u}_{k}, \ldots, \bar{u}_{j}\right)}(\omega)\right),
$$

where $\left(y_{r}^{k, x,\left(\bar{u}_{k}, \ldots, \bar{u}_{j}\right)}\right)_{r=k}^{j+1}$ satisfies the first equation in 8 for $\bar{u}_{k}, \ldots, \bar{u}_{j}$, and $y_{k}^{k, x,\left(\bar{u}_{k}, \ldots, \bar{u}_{j}\right)}=x$. Since the function $u_{j+1}$ is measurable and bounded, and $y_{j+1}^{k, x,\left(\bar{u}_{k}, \ldots, \bar{u}_{j}\right)}$ is measurable with respect to $\mathcal{F}_{j+1}^{k}$, we obtain that $\bar{u}=\left(\bar{u}_{k}, \ldots, \bar{u}_{N-1}\right) \in \mathcal{U}_{k}^{h}$. Using the same ideas as in (44)-(45), and the assumption $V_{j}^{h} \equiv S_{j}^{h}$, for all $k<j \leq N-1$, we get that

$$
\begin{aligned}
J_{k}^{h}(x, \bar{u}) & =h \ell\left(t_{k}, x, \bar{u}_{k}\right)+\mathbb{E}\left[h \sum_{j=k+1}^{N-1} \ell\left(t_{j}, y_{j}^{k, x, \bar{u}}, u_{j}\left(y_{j}^{k, x, \bar{u}}\right)\right)+g\left(y_{N}^{k, x, \bar{u}}\right)\right] \\
& =h \ell\left(t_{k}, x, \bar{u}_{k}\right)+\mathbb{E}\left[h \sum_{j=k+1}^{N-1} \ell\left(t_{j}, y_{j}^{k, x, \bar{u}}, u_{j}\left(y_{j}^{k, x, \bar{u}}\right)\right)+V_{N}^{h}\left(y_{N}^{k, x, \bar{u}}\right)\right] \\
& \leq h \ell\left(t_{k}, x, \bar{u}_{k}\right)+\mathbb{E}\left[h \sum_{j=k+1}^{N-2} \ell\left(t_{j}, y_{j}^{k, x, \bar{u}}, u_{j}\left(y_{j}^{k, x, \bar{u}}\right)\right)+S_{N-1}^{h}\left(y_{N-1}^{k, x, \bar{u}}\right)+\varepsilon\right] \\
& =h \ell\left(t_{k}, x, \bar{u}_{k}\right)+\mathbb{E}\left[h \sum_{j=k+1}^{N-2} \ell\left(t_{j}, y_{j}^{k, x, \bar{u}}, u_{j}\left(y_{j}^{k, x, \bar{u}}\right)\right)+V_{N-1}^{h}\left(y_{N-1}^{k, x, \bar{u}}\right)+\varepsilon\right] \\
& \leq h \ell\left(t_{k}, x, \bar{u}_{k}\right)+\mathbb{E}\left[V_{k+1}^{h}\left(y_{k+1}^{k, x, \bar{u}}\right)\right]+(N-1) \varepsilon \\
& \leq S_{k}^{h}(x)+N \varepsilon,
\end{aligned}
$$

where the inequality in the third line above is obtained by using (52). We conclude that,

$$
V_{k}^{h}(x) \leq J_{k}^{h}(x, \bar{u}) \leq S_{k}^{h}(x)+N \varepsilon \text {. }
$$

Since $\varepsilon>0$ is arbitrary, we obtain

from which the result follows.

$$
V_{k}^{h}(x) \leq S_{k}^{h}(x)
$$

The following remark will be used in the proof of the main result of Section 5 .

Remark 3.5. Given $k \in\{0, \ldots, N-1\}$, we introduce the following sets of controls,

$$
\left\{\begin{array}{l}
\overline{\mathcal{U}}_{k}^{h}:=\left\{u \in \Pi_{j=k}^{N-1} L_{\mathcal{F}_{j}^{0}}^{2}: u_{j} \in U_{a d}, \mathbb{P} \text {-a.s. } \forall j=k, \ldots, N-1\right\} \\
\overline{\mathcal{U}}_{k}^{1}:=\left\{u \in \Pi_{j=k}^{N-1} L_{\mathcal{F}_{t_{j}}^{0}}^{2}: u_{j} \in U_{a d}, \mathbb{P} \text {-a.s. } \forall j=k, \ldots, N-1\right\}, \\
\overline{\mathcal{U}}_{k}^{2}:=\left\{u \in \Pi_{j=k}^{N-1} L_{\mathcal{F}_{t_{j}}^{t_{k}}}^{2}: u_{j} \in U_{a d}, \mathbb{P} \text {-a.s. } \forall j=k, \ldots, N-1\right\},
\end{array}\right.
$$

and the associated value functions,

$$
\left\{\begin{array}{lll}
\bar{V}_{N}^{h}(x):=g(x), & \bar{V}_{k}^{h}(x):=\inf _{u \in \overline{\mathcal{U}}_{k}^{h}} J_{k}^{h}(x, u), & k=0, \ldots, N-1, \\
\bar{V}_{N}^{h, 1}(x):=g(x), & \bar{V}_{k}^{h, 1}(x):=\inf _{u \in \overline{\mathcal{U}}_{k}^{1}} J_{k}^{h}(x, u), & k=0, \ldots, N-1, \\
\bar{V}_{N}^{h, 2}(x):=g(x), & \bar{V}_{k}^{h, 2}(x):=\inf _{u \in \overline{\mathcal{U}}_{k}^{2}} J_{k}^{h}(x, u), & k=0, \ldots, N-1 .
\end{array}\right.
$$

We can observe that all the results of the current section including the DPP, remain true if we deal with any of the sets in (57). Indeed, the proofs are based in the fact that the processes are adapted to the given filtration and the increments of Brownian motions are independent. Since $\bar{V}_{N}^{h}(x)=\bar{V}_{N}^{h, 1}(x)=\bar{V}_{N}^{h, 2}(x)=V_{N}^{h}(x)=g(x)$, and $\left\{\bar{V}_{k}^{h}\right\},\left\{\bar{V}_{k}^{h, 1}\right\},\left\{\bar{V}_{k}^{h, 2}\right\}$ and $\left\{V_{k}^{h}\right\}$ satisfy 40 , we have

$$
\bar{V}_{k}^{h}(x)=\bar{V}_{k}^{h, 1}(x)=\bar{V}_{k}^{h, 2}(x)=V_{k}^{h}(x), \quad \forall k=0, \ldots, N, \quad \forall x \in \mathbb{R}^{n} .
$$




\subsection{Feedback optimal control}

The aim of this section is to prove that there exists a feedback optimal control for $\left(P_{k, x}^{h}\right)$. For notational convenience we define for all $0 \leq k \leq N-1$, the function $F^{k}: \mathbb{R}^{n} \times U_{a d} \rightarrow \mathbb{R}$ as

$$
F^{k}(x, u):=h \ell\left(t_{k}, x, u\right)+\mathbb{E}\left[V_{k+1}^{h}\left(x+h f\left(t_{k}, x, u\right)+\sigma\left(t_{k}, x, u\right) \Delta W_{k+1}\right)\right] .
$$

Then, by the DPP, for all $x \in \mathbb{R}^{n}$ and $k=0, \ldots, N-1$, we have,

$$
V_{k}^{h}(x)=\inf _{u \in U_{a d}} F^{k}(x, u) \quad \text { and } \quad V_{N}^{h}(x)=g(x) .
$$

Based on a measurable selection theorem due to Schäl [28, Theorem 5.3.1], we can prove the following result.

Proposition 3.6. Under the above assumptions, for all $k=0, \ldots, N-1$ there exists a measurable function $\bar{u}^{k}: \mathbb{R}^{n} \rightarrow U_{a d}$ such that

$$
F^{k}\left(x, \bar{u}^{k}(x)\right)=V_{k}^{h}(x)
$$

for all $x \in \mathbb{R}^{n}$.

Proof. Arguing as in the proof of Lemma 3.3. it is easy to check that $(x, u) \mapsto F^{k}(x, u)$ is continuous. Since $U_{a d}$ is compact we can apply [28, Theorem 5.3.1]. The result follows.

Remark 3.7. As a corollary of the previous results and the DPP, in this discrete framework, we always have a discrete time feedback (also called Markov) optimal control. Indeed, the sequence of measurable functions $\bar{u}_{0}, \ldots, \bar{u}_{N-1}$ given by the previous proposition, defines the optimal control $\bar{u}=\left(\bar{u}^{0}\left(y_{0}\right), \bar{u}^{1}\left(y_{1}\right), \ldots, \bar{u}^{N-1}\left(y_{N-1}\right)\right)$, where $\left(y_{0}, \ldots, y_{N}\right)$ is defined recursively as

$$
\left\{\begin{array}{l}
y_{k+1}=y_{k}+h f\left(t_{k}, y_{k}, \bar{u}^{k}\left(y_{k}\right)\right)+\sigma\left(t_{k}, y_{k}, \bar{u}^{k}\left(y_{k}\right)\right) \Delta W_{k+1} \quad \forall k=0, \ldots, N-1, \\
y_{0}=x
\end{array}\right.
$$

Let us point out an interesting phenomenon, not underlined enough in the literature, which shows the power of the DPP. In the continuous time case it is well known (see e.g. [13. Chapter VI] and [14. Chapters 3 and 4]) that if the Hamilton-Jacobi-Bellman equation associated with the stochastic control problem, which is a consequence of the DPP in continuous time, admits a solution $v$ which is regular enough, then we can construct a feedback optimal control. This is known as a verification result and, under standard assumptions, usually holds when $\sigma$ does not depend on $u$ and, setting $a=\sigma \sigma^{\top}$, we have that

$$
\sum_{1 \leq i, j \leq n} a_{i, j}(x, t) \xi_{i} \xi_{j} \geq c|\xi|^{2} \quad \forall \xi \in \mathbb{R}^{d}
$$

for some $c>0$. In particular, if we fix $(t, x) \in\left[0, T\left[\times \mathbb{R}^{n}\right.\right.$, we get the existence of an optimal feedback policy for the problem associated with $V(t, x)$. The main feature of this analysis is that existence of an optimum is obtained without some usual convexity assumptions required in the strong formulation (see e.g. 30, Chapter 2 , Section 5.2]). On the other hand, as we have just seen, for the problem obtained by discretizing the time variable we always have the existence of a feedback control without any extra assumption. This is still an infinite dimensional problem for which existence does not follow by standard methods, but it is a consequence of the DPP. 


\section{Other Regularity PROPERTIES of the VAlue FunCtion}

In this section we prove some regularity properties of the value function of the continuous and the discrete problems. Some of them will be used in the proof of our main result in the next section and the others are interesting by themselves.

In the following result we show the local (in space) Hölder continuity in time for $V$ as well as an analogous result for its discrete version $\left\{V_{k}^{h} ; k=0, \ldots, N\right\}$ defined in 10 . The former result is classical, see e.g. 29 , Section 3.4] and 30, Chapter 4, Proposition 3.1]). However, for the sake of completeness, we prove here a version adapted to our assumptions.

In the statement of the following result we use the r.h.s. of (2) (respectively (9)) to extend $J^{s, x}(\cdot)$ (respectively $\left.J_{k}^{h}(x, \cdot)\right)$ to $\mathcal{U}_{a d}^{0}$ (respectively $\left.\mathcal{U}_{0}^{h}\right)$.

Theorem 4.1. Under assumptions (H1) and (H2), there exists $C>0$ (independent of $h$ ), such that for all $x \in \mathbb{R}^{n}, u \in \mathcal{U}_{a d}^{0}$ and $s, t \in[0, T]$,

$$
\left|J^{s, x}(u)-J^{t, x}(u)\right| \leq C\left[1+|x|^{2}\right]|s-t|^{\frac{1}{2}},
$$

and for all $u \in \mathcal{U}_{0}^{h}$ and $r, k=0, \ldots, N$,

$$
\left|J_{r}^{h}(x, u)-J_{k}^{h}(x, u)\right| \leq C\left[1+|x|^{2}\right]|k-r|^{\frac{1}{2}} h^{\frac{1}{2}} .
$$

As a consequence,

$$
|V(s, x)-V(t, x)| \leq C\left[1+|x|^{2}\right]|s-t|^{\frac{1}{2}} \quad \forall x \in \mathbb{R}^{n}, \quad s, t \in[0, T],
$$

and

$$
\left|V_{r}^{h}(x)-V_{k}^{h}(x)\right| \leq C\left[1+|x|^{2}\right]|k-r|^{\frac{1}{2}} h^{\frac{1}{2}} \quad \forall x \in \mathbb{R}^{n}, \quad r, k=0, \ldots, N .
$$

Proof. First of all note that for all $s \in[0, T]$ and $x \in \mathbb{R}^{n}$, we have

$$
V(s, x)=\inf _{u \in \mathcal{U}_{a d}^{0}} J^{s, x}(u) .
$$

Indeed, it is clear that $V(s, x) \geq \inf _{u \in \mathcal{U}_{a d}^{0}} J^{s, x}(u)$. On the other hand, if $u \in \mathcal{U}_{a d}^{0}$, then for all $s \leq t \leq T$ the function $u(t)$ is $\mathcal{F}_{t}^{0}$-measurable, and so there exists a measurable map $u_{t}\left(\left(\omega_{r}\right)_{0 \leq r \leq s},\left(\omega_{r}-\omega_{s}\right)_{s \leq r \leq t}\right)$ such that $u(t, \omega)=u_{t}\left(\left(W_{r}(\omega)\right)_{0 \leq r \leq s},\left(W_{r}(\omega)-W_{s}(\omega)\right)_{s \leq r \leq t}\right)$, P-a.s. ( [1]). Then if we fix $\left(\omega_{r}\right)_{0 \leq r \leq s}$ we can define the function

$$
\hat{u}_{t}\left(\left(\omega_{r}\right)_{0 \leq r \leq s}\right): \omega \in \Omega \mapsto u_{t}\left(\left(\omega_{r}\right)_{0 \leq r \leq s},\left(W_{r}(\omega)-W_{s}(\omega)\right)_{s \leq r \leq t}\right),
$$

which belongs to $\mathcal{F}_{t}^{s}$. By the independence of the increments of Brownian motions, we obtain the converse inequality (see, e.g. [5, Remark 5.2]).

Without loss of generality, assume that $0 \leq s \leq t \leq T$. We consider a fixed initial state $x$ and a control $u \in \mathcal{U}_{a d}^{0}$. For simplicity we denote $y^{s}:=y_{u}^{s, x}, y^{t}:=y_{u}^{t, x}$ and for $t \leq r \leq T$, and $\varphi=f, \sigma$,

$$
\Delta y(r):=y^{s}(r)-y^{t}(r) \text { and } \Delta \varphi(r)=\varphi\left(r, y^{s}(r), u(r)\right)-\varphi\left(r, y^{t}(r), u(r)\right) .
$$

Then, we obtain for $t \leq r \leq T$

$$
\begin{aligned}
\Delta y(r)= & \int_{s}^{t} f\left(\tau, y^{s}(\tau), u(\tau)\right) \mathrm{d} \tau+\int_{s}^{t} \sigma\left(\tau, y^{s}(\tau), u(\tau)\right) \mathrm{d} W(\tau) \\
& +\int_{t}^{r} \Delta f(\tau) \mathrm{d} \tau+\int_{t}^{r} \Delta \sigma(\tau) \mathrm{d} W(\tau) .
\end{aligned}
$$


By assumption (H1), the Cauchy-Schwarz inequality and the Itô isometry we have

$$
\begin{aligned}
\mathbb{E}\left[|\Delta y(r)|^{2}\right] \leq & 4[|s-t|+1] \int_{s}^{t} 3 L^{2}\left(1+\mathbb{E}\left[\left|y^{s}(\tau)\right|^{2}\right]+\mathbb{E}\left[|u(\tau)|^{2}\right]\right) \mathrm{d} \tau \\
& +4[|s-t|+1] \int_{t}^{r} L^{2} \mathbb{E}\left[|\Delta y(\tau)|^{2}\right] \mathrm{d} \tau .
\end{aligned}
$$

Since the set $U_{a d}$ is compact, by the Grönwall lemma [10 and [24, Proposition 2.1] there exists $C_{0}>0$ such that

$$
\sup _{t \leq r \leq T} \mathbb{E}\left[|\Delta y(r)|^{2}\right] \leq C_{0}\left[1+|x|^{2}\right]|s-t| .
$$

Now we compare $J^{s, x}(u)$ and $J^{t, x}(u)$, we denote $\Delta \ell(r):=\ell\left(r, y^{s}(r), u(r)\right)-\ell\left(r, y^{t}(r), u(r)\right)$ and $\Delta g=g\left(y^{s}(T)\right)-$ $g\left(y^{t}(T)\right)$. We have

$$
\left|J^{s, x}(u)-J^{t, x}(u)\right| \leq \mathbb{E} \int_{s}^{t}\left|\ell\left(r, y^{s}(r), u(r)\right)\right| \mathrm{d} r+\mathbb{E} \int_{t}^{T}|\Delta \ell(r)| \mathrm{d} r+\mathbb{E}|\Delta g| .
$$

By assumption (H2) we obtain,

$$
\mathbb{E} \int_{s}^{t}\left|\ell\left(r, y^{s}(r), u(r)\right)\right| \mathrm{d} r \leq 3 L \int_{s}^{t}\left[\mathbb{E}\left[\left|y^{s}(r)\right|^{2}\right]+\mathbb{E}\left[|u(r)|^{2}\right]+1\right] \mathrm{d} r,
$$

and since $U_{a d}$ is bounded, again by [24. Proposition 2.1] there exists $C_{1}>0$ such that

$$
\mathbb{E} \int_{s}^{t}\left|\ell\left(r, y^{s}(r), u(r)\right)\right| \mathrm{d} r \leq C_{1}\left[1+|x|^{2}\right]|s-t| .
$$

On the other hand, for the last two terms we obtain,

$$
\begin{aligned}
\mathbb{E}[|\Delta \ell(r)|] & \leq \mathbb{E}\left[\int_{0}^{1}\left|\ell_{y}\left(r, y^{t}(r)+\xi \Delta y(r), u(r)\right) \Delta y(r)\right| \mathrm{d} \xi\right] \\
& \leq \mathbb{E}\left[\int_{0}^{1}\left[L\left(1+\left|y^{t}(r)\right|+\xi|\Delta y(r)|+|u(r)|\right)|\Delta y(r)|\right] \mathrm{d} \xi\right] \\
& \leq L \mathbb{E}\left[\left[1+\left|y^{s}(r)\right|+|\Delta y(r)|+|u(r)|\right]|\Delta y(r)|\right] .
\end{aligned}
$$

By the Cauchy-Schwarz inequality, Remark 2.1] the compactness of $U_{a d}$ and 773 , we deduce that there exists $C_{2}>0$ such that

$$
\mathbb{E}[|\Delta \ell(r)|] \leq C_{2}\left[1+|x|^{2}\right]|s-t|^{\frac{1}{2}}
$$

An analogous estimate holds for $\Delta g$ and so the result follows.

In the case of the discrete value function, Remark 3.5 implies that for all $x \in \mathbb{R}^{n}$,

$$
V_{k}^{h}(x)=\inf _{u \in \mathcal{U}_{0}^{h}} \mathbb{E}\left[h \sum_{j=k}^{N-1} \ell\left(t_{j}, y_{j}^{k, x, u}, u_{j}\right)+g\left(y_{N}^{k, x, u}\right)\right], \quad k \in\{0, \ldots, N\} .
$$

Let $u=\left(u_{j}\right) \in \mathcal{U}_{0}^{h}$ be a given control and $r, k \in\{0, \ldots, N-1\}$ such that $r<k$. Let us set

$$
\Delta y_{j}=y_{j}^{r, x, u}-y_{j}^{k, x, u}, \Delta \varphi_{j}=\varphi\left(t_{j}, y_{j}^{r, x, u}, u_{j}\right)-\varphi\left(t_{j}, y_{j}^{k, x, u}, u_{j}\right) \text { and } \Delta g=g\left(y_{N}^{r, x, u}\right)-g\left(y_{N}^{k, x, u}\right),
$$

for $\varphi=f, \sigma, \ell$ and $j=k, \ldots, N-1$. Then

$$
\Delta y_{j+1}=\Delta y_{j}+h \Delta f_{j}+\Delta \sigma_{j} \Delta W_{j+1}
$$


Following the techniques in Subsection 2.4 there exists $C_{3}>0$ such that

$$
\mathbb{E}\left[\left|\Delta y_{j+1}\right|^{2}\right] \leq\left(1+C_{3} h\right) \mathbb{E}\left[\left|\Delta y_{j}\right|^{2}\right] \leq e^{C_{3} T} \mathbb{E}\left[\left|\Delta y_{k}\right|^{2}\right]
$$

By the definition, we have $y_{k}^{k, x, u}=x$, and hence,

$$
\Delta y_{k}=y_{k}^{r, x, u}-x=h \sum_{j=r}^{k-1} f\left(t_{j}, y_{j}^{r, x, u}, u_{j}\right)+\sum_{j=r}^{k-1}\left[\sigma\left(t_{j}, y_{j}^{r, x, u}, u_{j}\right) \Delta W_{j+1}\right]
$$

Since $U_{a d}$ is compact, by the independence of the increments of the Brownian motion and (H1), there exist $C_{4}>0$ and $C_{5}>0$ such that

$$
\begin{aligned}
\mathbb{E}\left[\left|\Delta y_{k}\right|^{2}\right] & \leq C_{4}\left[h^{2}(k-r) \sum_{j=r}^{k-1} \mathbb{E}\left[\left|f\left(t_{j}, y_{j}^{r, x, u}, u_{j}\right)\right|^{2}\right]+h \sum_{j=r}^{k-1} \mathbb{E}\left[\left|\sigma\left(t_{j}, y_{j}^{r, x, u}, u_{j}\right)\right|^{2}\right]\right] \\
& \leq C_{5} h|k-r|\left[1+\max _{j=r, \ldots, k-1} \mathbb{E}\left[\left|y_{j}^{r, x, u}\right|^{2}\right]\right] \\
& \leq C_{6} h|k-r|\left[1+|x|^{2}\right],
\end{aligned}
$$

where the last inequality holds by Lemma 2.2. We have

$$
\left|J_{r}^{h}(x, u)-J_{k}^{h}(x, u)\right|=\left|h \sum_{j=r}^{k-1} \mathbb{E}\left[\ell\left(t_{j}, y_{j}^{r, x, u}, u_{j}\right)\right]+h \sum_{j=k}^{N-1} \mathbb{E}\left[\Delta \ell_{j}\right]+\mathbb{E}[\Delta g]\right|,
$$

and also,

$$
\left|V_{r}^{h}(x)-V_{k}^{h}(x)\right| \leq \sup _{u \in \mathcal{U}_{0}^{h}}\left|J_{r}^{h}(x, u)-J_{k}^{h}(x, u)\right| .
$$

By (H2), Lemma 2.2 and the compactness of $U_{a d}$, there exists $C_{7}>0$ such that

$$
h\left|\sum_{j=r}^{k-1} \mathbb{E}\left[\ell\left(t_{j}, y_{j}^{r, x, u}, u_{j}\right)\right]\right| \leq h \sum_{j=r}^{k-1} L \mathbb{E}\left[1+\left|y_{j}^{r, x, u}\right|+\left|u_{j}\right|\right]^{2} \leq h|k-r| C_{7}\left[1+|x|^{2}\right] .
$$

On the other hand, as in (77), by Lemma 2.2 and 84 , we obtain the existence of $C_{8}>0$ such that

$$
\mathbb{E}\left[\Delta \ell_{j}\right] \leq C_{8}\left[1+|x|^{2}\right] h^{\frac{1}{2}}|k-r|^{\frac{1}{2}}
$$

and a similar estimate holds for $\mathbb{E}[\Delta g]$. Therefore, combining 85 - 88 we get the result.

Our aim now is to study the semiconcavity of $V$ and of its discrete version $\left\{V_{k}^{h} ; k=0, \ldots, N\right\}$. Recall that $\varphi: \mathbb{R}^{n} \rightarrow \mathbb{R}$ is locally semi-concave in $\mathbb{R}^{n}$ if for all $x \in \mathbb{R}^{n}$ and $\delta>0$, there exists a constant $K_{x, \delta}>0$ such that, for all $y \in B_{\delta}(x):=\left\{z \in \mathbb{R}^{n}:|z-x|<\delta\right\}$ and $\lambda \in[0,1]$

$$
\lambda \varphi(x)+(1-\lambda) \varphi(y)-\varphi(\lambda x+(1-\lambda) y) \leq K_{x, \delta} \lambda(1-\lambda)|x-y|^{2} .
$$

We will need the following additional assumptions:

(H3) There exists $K>0$ such that $g$ is semi-concave with constant $K$ and $\ell$ is also semi-concave with constant $K$, uniformly in $[0, T] \times U_{a d}$, i.e. for all $y, \bar{y} \in \mathbb{R}^{n}$,

$$
\lambda \ell(t, y, u)+(1-\lambda) \ell(t, \bar{y}, u)-\ell(t, \lambda y+(1-\lambda) \bar{y}, u) \leq K \lambda(1-\lambda)|y-\bar{y}|^{2}, \quad \forall \lambda \in[0,1],
$$

and

$$
\lambda g(y)+(1-\lambda) g(\bar{y})-g(\lambda y+(1-\lambda) \bar{y}) \leq K \lambda(1-\lambda)|y-\bar{y}|^{2}, \quad \forall \lambda \in[0,1] .
$$


(H4) For $\varphi=f, \sigma$, and for almost all $t \in[0, T]$, the map $(y, u) \mapsto \varphi(t, y, u)$ is $C^{2}$ and there exists a constant $L$ such that for all $y \in \mathbb{R}^{n}$ and $u \in U_{a d}$,

$$
\left|\varphi_{y y}(t, y, u)\right| \leq L
$$

Under these additional assumption we prove now a local version of 89 for $V$ and $\left\{V_{k}^{h} ; k=0, \ldots, N\right\}$. The following proof is similar to [30, Proposition 4.5, p.187].

Theorem 4.2. Under assumptions $(\mathbf{H 1})-(\mathbf{H 4})$, the functions $V$ and $V_{k}^{h}$ are locally semi-concave with respect to the space variable, i.e. for all $\bar{x} \in \mathbb{R}^{n}, \delta>0, s \in[0, T]$ and $k=0, \ldots, N, V(s, \cdot)$ and $V_{k}^{h}(\cdot)$ satisfy (89) with constants $K_{\bar{x}, \delta}>0$ which are independent of $s$ and $k$, respectively.

Proof. Let $x, \bar{x} \in \mathbb{R}^{n}, \lambda \in[0,1]$ and define $x^{\lambda}:=\lambda x+(1-\lambda) \bar{x}$. For any $\varepsilon>0$, there exists $u_{\varepsilon} \in \mathcal{U}_{a d}^{s}$ such that

$$
J^{s, x^{\lambda}}\left(u_{\varepsilon}\right)-\varepsilon<V\left(s, x^{\lambda}\right) \text {. }
$$

For notational convenience, we denote $y^{\xi}(t)=y_{u_{\varepsilon}}^{s, \xi}(t), \ell\left(y^{\xi}(t)\right)=\ell\left(t, y_{u_{\varepsilon}}^{s, \xi}(t), u_{\varepsilon}(t)\right)$ and $g\left(y^{\xi}\right)=g\left(y_{u_{\varepsilon}}^{s, \xi}(T)\right)$ for $\xi=x, \bar{x}, x^{\lambda}$. Then, we have

$$
\begin{aligned}
\lambda V(s, x)+(1-\lambda) V(s, \bar{x})-V\left(s, x^{\lambda}\right) \leq & \lambda J^{s, x}\left(u_{\varepsilon}\right)+(1-\lambda) J^{s, \bar{x}}\left(u_{\varepsilon}\right)-J^{s, x^{\lambda}}\left(u_{\varepsilon}\right)+\varepsilon \\
\leq & \mathbb{E}\left[\int_{s}^{T}\left[\lambda \ell\left(y^{x}\right)+(1-\lambda) \ell\left(y^{\bar{x}}\right)-\ell\left(y^{x^{\lambda}}\right)\right] \mathrm{d} t\right] \\
& +\mathbb{E}\left[\lambda g\left(y^{x}\right)+(1-\lambda) g\left(y^{\bar{x}}\right)-g\left(y^{x^{\lambda}}\right)\right]+\varepsilon .
\end{aligned}
$$

By the semi-concavity assumption (H3) we obtain,

$$
\begin{aligned}
\mathbb{E}\left[\int_{s}^{T}\left[\lambda \ell\left(y^{x}\right)+(1-\lambda) \ell\left(y^{\bar{x}}\right)-\ell\left(y^{x^{\lambda}}\right)\right] \mathrm{d} t\right]= & \mathbb{E}\left[\int_{s}^{T}\left[\lambda \ell\left(y^{x}\right)+(1-\lambda) \ell\left(y^{\bar{x}}\right)-\ell\left(\lambda y^{x}+(1-\lambda) y^{\bar{x}}\right)\right] \mathrm{d} t\right] \\
& +\mathbb{E}\left[\int_{s}^{T}\left[\ell\left(\lambda y^{x}+(1-\lambda) y^{\bar{x}}\right)-\ell\left(y^{x^{\lambda}}\right)\right] \mathrm{d} t\right] \\
\leq & K \lambda(1-\lambda) T \mathbb{E}\left[\sup _{t \in[s, T]}\left|y^{x}(t)-y^{\bar{x}}(t)\right|^{2}\right] \\
& +\mathbb{E}\left[\int_{s}^{T}\left[\ell\left(\lambda y^{x}+(1-\lambda) y^{\bar{x}}\right)-\ell\left(y^{x^{\lambda}}\right)\right] \mathrm{d} t\right] .
\end{aligned}
$$

By (17), there exists $C_{0}$ such that

$$
\mathbb{E}\left[\sup _{t \in[s, T]}\left|y^{x}(t)-y^{\bar{x}}(t)\right|^{2}\right] \leq C_{0}|x-\bar{x}|^{2} .
$$

Now, define $\Delta y(t):=\lambda y^{x}(t)+(1-\lambda) y^{\bar{x}}(t)-y^{x^{\lambda}}(t)$ for all $t \in[s, T]$. By (H2), and the compactness of $U_{a d}$, there exists $C_{1}>0$ such that

$$
\begin{aligned}
\left|\ell\left(\lambda y^{x}(t)+(1-\lambda) y^{\bar{x}}(t)\right)-\ell\left(y^{x^{\lambda}}(t)\right)\right| & \leq \int_{0}^{1}\left|\ell_{y}\left(y^{x^{\lambda}}(t)+\xi \Delta y(t)\right)\right||\Delta y(t)| \mathrm{d} \xi \\
& \leq C_{1}\left[1+\left|y^{x^{\lambda}}(t)\right|+|\Delta y(t)|\right]|\Delta y(t)| .
\end{aligned}
$$

We can obtain a similar estimate for $g$. By $(\mathbf{H} 4)$, there exists $C_{2}>0$ such that

$$
\mathbb{E}\left[\sup _{t \in[s, T]}|\Delta y(t)|^{2}\right] \leq C_{2} \lambda^{2}(1-\lambda)^{2}|x-\bar{x}|^{4} .
$$


Now, returning to (97), by the Cauchy-Schwartz inequality, (17) and the above equation, there exists $C_{3}>0$ such that

$$
\begin{aligned}
\mathbb{E}\left[\left|y^{x^{\lambda}}(t)\right||\Delta y(t)|\right] & \leq\left(\mathbb{E}\left|y^{x^{\lambda}}(t)\right|^{2}\right)^{\frac{1}{2}}\left(\mathbb{E}|\Delta y(t)|^{2}\right)^{\frac{1}{2}} \\
& \leq C_{3}\left[1+\left|x^{\lambda}\right|\right] \lambda(1-\lambda)|x-\bar{x}|^{2} \\
& \leq C_{3}[1+|\bar{x}|+|x-\bar{x}|] \lambda(1-\lambda)|x-\bar{x}|^{2} .
\end{aligned}
$$

Since $|x-\bar{x}|^{4} \leq \delta^{2}|x-\bar{x}|^{2}$, for all $x \in B_{\delta}(\bar{x})$, combining (95)-(99), we can complete the proof of 89 for $V(s, \cdot)$.

Now, for the discrete value, we follow similar arguments. There exists $u_{\varepsilon} \in \mathcal{U}_{k}^{h}$ such that

$$
J_{k}^{h}\left(x^{\lambda}, u_{\varepsilon}\right)-\varepsilon \leq V_{k}^{h}\left(x^{\lambda}\right) .
$$

Denoting $y_{j}^{\xi}=y_{j}^{k, \xi, u_{\varepsilon}}$ and $\ell\left(y_{j}^{\xi}\right)=\ell\left(t_{j}, y_{j}^{\xi}, u_{\varepsilon, j}\right)$ for $\xi=x, \bar{x}, x^{\lambda}$ and $j=k, \ldots, N$, we have

$$
\begin{aligned}
\lambda V_{k}^{h}(x)+(1-\lambda) V_{k}^{h}(\bar{x})-V_{k}^{h}\left(x^{\lambda}\right) \leq & \lambda J_{k}^{h}\left(x, u_{\varepsilon}\right)+(1-\lambda) J_{k}^{h}\left(\bar{x}, u_{\varepsilon}\right)-J_{k}^{h}\left(x^{\lambda}, u_{\varepsilon}\right)+\varepsilon \\
\leq & \mathbb{E}\left[h \sum_{j=k}^{N-1}\left[\lambda \ell\left(y_{j}^{x}\right)+(1-\lambda) \ell\left(y_{j}^{\bar{x}}\right)-\ell\left(y_{j}^{x^{\lambda}}\right)\right]\right] \\
& +\mathbb{E}\left[\lambda g\left(y_{N}^{x}\right)+(1-\lambda) g\left(y_{N}^{\bar{x}}\right)-g\left(y_{N}^{x^{\lambda}}\right)\right]+\varepsilon .
\end{aligned}
$$

As in (95), by the semi-concavity assumption (H3) we obtain

$$
\begin{aligned}
\mathbb{E}\left[h \sum_{j=k}^{N-1}\left[\lambda \ell\left(y_{j}^{x}\right)+(1-\lambda) \ell\left(y_{j}^{\bar{x}}\right)-\ell\left(y_{j}^{x^{\lambda}}\right)\right]\right] \leq & K \lambda(1-\lambda) T \max _{j=k, \ldots, N} \mathbb{E}\left[\left|y_{j}^{x}-y_{j}^{\bar{x}}\right|^{2}\right] \\
& +\mathbb{E}\left[h \sum_{j=k}^{N-1}\left[\ell\left(\lambda y_{j}^{x}+(1-\lambda) y_{j}^{\bar{x}}\right)-\ell\left(y_{j}^{x^{\lambda}}\right)\right]\right] .
\end{aligned}
$$

By Lemma 2.3, there exists $C_{0}>0$ such that

$$
\max _{j=k, \ldots, N} \mathbb{E}\left[\left|y_{j}^{x}-y_{j}^{\bar{x}}\right|^{2}\right] \leq C_{0}|x-\bar{x}|^{2} .
$$

In order to estimate the last term in 102 we set $\Delta y_{j}:=\lambda y_{j}^{x}+(1-\lambda) y_{j}^{\bar{x}}-y_{j}^{x^{\lambda}}$. By (H2) and the compactness of $U_{a d}$, there exists $C_{1}>0$ such that

$$
\begin{aligned}
\mathbb{E}\left[\ell\left(\lambda y_{j}^{x}+(1-\lambda) y_{j}^{\bar{x}}\right)-\ell\left(y_{j}^{x^{\lambda}}\right)\right] & \leq \mathbb{E}\left[\int_{0}^{1}\left|\ell_{y}\left(y_{j}^{x^{\lambda}}+\xi \Delta y_{j}\right)\right|\left|\Delta y_{j}\right| \mathrm{d} \xi\right] \\
& \leq C_{1} \mathbb{E}\left[\left[1+\left|y_{j}^{x^{\lambda}}\right|+\left|\Delta y_{j}\right|\right]\left|\Delta y_{j}\right|\right] .
\end{aligned}
$$

We have

$$
\begin{aligned}
\Delta y_{j+1}= & \Delta y_{j}+h\left[f\left(\lambda y_{j}^{x}+(1-\lambda) y_{j}^{\bar{x}}\right)-f\left(y_{j}^{x^{\lambda}}\right)\right]+\left[\sigma\left(\lambda y_{j}^{x}+(1-\lambda) y_{j}^{\bar{x}}\right)-\sigma\left(y_{j}^{x^{\lambda}}\right)\right] \Delta W_{j+1} \\
& +h\left[\lambda f\left(y_{j}^{x}\right)+(1-\lambda) f\left(y_{j}^{\bar{x}}\right)-f\left(\lambda y_{j}^{x}+(1-\lambda) y_{j}^{\bar{x}}\right)\right] \\
& +\left[\lambda \sigma\left(y_{j}^{x}\right)+(1-\lambda) \sigma\left(y_{j}^{\bar{x}}\right)-\sigma\left(\lambda y_{j}^{x}+(1-\lambda) y_{j}^{\bar{x}}\right)\right] \Delta W_{j+1} .
\end{aligned}
$$

By the Young's inequality and the Itô isometry, there exists $C_{2}>0$ such that,

$$
\begin{aligned}
\mathbb{E}\left[\left|\Delta y_{j+1}\right|^{2}\right] \leq & {\left[1+C_{2} h\right] \mathbb{E}\left[\left|\Delta y_{j}\right|^{2}\right]+C_{2} h \mathbb{E}\left[\left|f\left(\lambda y_{j}^{x}+(1-\lambda) y_{j}^{\bar{x}}\right)-f\left(y_{j}^{x^{\lambda}}\right)\right|^{2}\right] } \\
& +C_{2} h \mathbb{E}\left[\left|\sigma\left(\lambda y_{j}^{x}+(1-\lambda) y_{j}^{\bar{x}}\right)-\sigma\left(y_{j}^{x^{\lambda}}\right)\right|^{2}\right] \\
& +C_{2} h \mathbb{E}\left[\left|\lambda f\left(y_{j}^{x}\right)+(1-\lambda) f\left(y_{j}^{\bar{x}}\right)-f\left(\lambda y_{j}^{x}+(1-\lambda) y_{j}^{\bar{x}}\right)\right|^{2}\right] \\
& +C_{2} h \mathbb{E}\left[\left|\lambda \sigma\left(y_{j}^{x}\right)+(1-\lambda) \sigma\left(y_{j}^{\bar{x}}\right)-\sigma\left(\lambda y_{j}^{x}+(1-\lambda) y_{j}^{\bar{x}}\right)\right|^{2}\right] .
\end{aligned}
$$


By (H1), we obtain

$$
\mathbb{E}\left[\left|f\left(\lambda y_{j}^{x}+(1-\lambda) y_{j}^{\bar{x}}\right)-f\left(y_{j}^{x^{\lambda}}\right)\right|^{2}\right]+\mathbb{E}\left[\left|\sigma\left(\lambda y_{j}^{x}+(1-\lambda) y_{j}^{\bar{x}}\right)-\sigma\left(y_{j}^{x^{\lambda}}\right)\right|^{2}\right] \leq 2 L^{2} \mathbb{E}\left[\left|\Delta y_{j}\right|^{2}\right] .
$$

Now, for the last two terms in 106 we have,

$$
\begin{aligned}
\mid \lambda f\left(y_{j}^{x}\right)+(1-\lambda) f( & \left.y_{j}^{\bar{x}}\right)-f\left(\lambda y_{j}^{x}+(1-\lambda) y_{j}^{\bar{x}}\right) \mid \\
= & \mid \lambda \int_{0}^{1} f_{y}\left(\lambda y_{j}^{x}+(1-\lambda) y_{j}^{\bar{x}}+\xi(1-\lambda)\left(y_{j}^{x}-y_{j}^{\bar{x}}\right)\right)(1-\lambda)\left(y_{j}^{x}-y_{j}^{\bar{x}}\right) \mathrm{d} \xi \\
& +(1-\lambda) \int_{0}^{1} f_{y}\left(\lambda y_{j}^{x}+(1-\lambda) y_{j}^{\bar{x}}+\xi \lambda\left(y_{j}^{\bar{x}}-y_{j}^{x}\right)\right) \lambda\left(y_{j}^{\bar{x}}-y_{j}^{x}\right) \mathrm{d} \xi \mid \\
\leq & L \lambda(1-\lambda)\left|y_{j}^{x}-y_{j}^{\bar{x}}\right|^{2},
\end{aligned}
$$

where the last inequality holds by (H4). Analogous estimates are satisfied by $\sigma$. By Lemma 2.3, we can conclude that there exists $C_{3}>0$ and $C_{4}>0$ such that,

$$
\begin{aligned}
\mathbb{E}\left[\left|\Delta y_{j+1}\right|^{2}\right] & \leq\left[1+C_{3} h\right] \mathbb{E}\left[\left|\Delta y_{j}\right|^{2}\right]+C_{4} h \lambda^{2}(1-\lambda)^{2}|x-\bar{x}|^{4} \\
& \leq e^{C_{3} T} C_{4} \lambda^{2}(1-\lambda)^{2}|x-\bar{x}|^{4}
\end{aligned}
$$

By the Cauchy-Schwarz inequality, and the above inequality, there exists $C_{5}>0$ such that

$$
\sup _{j=k, \ldots, N} \mathbb{E}\left[\left|\Delta y_{j}\right|\right] \leq C_{5} \lambda(1-\lambda)|x-\bar{x}|^{2} .
$$

In order to estimate (104), by the Cauchy-Schwarz inequality, Lemma 2.2 and the previous bounds, there exists $C_{6}>0$ such that,

$$
\begin{aligned}
\mathbb{E}\left[\left|y_{j}^{x^{\lambda}}\right|\left|\Delta y_{j}\right|\right] & \leq\left(\mathbb{E}\left[\left|y_{j}^{x^{\lambda}}\right|^{2}\right]\right)^{\frac{1}{2}}\left(\mathbb{E}\left|\Delta y_{j}\right|^{2}\right)^{\frac{1}{2}} \\
& \leq C_{6}\left[1+\left|x^{\lambda}\right|\right] \lambda(1-\lambda)|x-\bar{x}|^{2} \\
& \leq C_{6}[1+|\bar{x}|+|x-\bar{x}|] \lambda(1-\lambda)|x-\bar{x}|^{2} .
\end{aligned}
$$

Since $|x-\bar{x}|^{4} \leq \delta^{2}|x-\bar{x}|^{2}$, for all $x \in B_{\delta}(\bar{x})$, combining (104), 110) and 1111, we deduce that there exists $C_{\bar{x}, \delta}>0$, which depends on $\bar{x}$ and $\delta$, such that

$$
\mathbb{E}\left[h \sum_{j=k}^{N-1}\left[\lambda \ell\left(y_{j}^{x}\right)+(1-\lambda) \ell\left(y_{j}^{\bar{x}}\right)-\ell\left(y_{j}^{x^{\lambda}}\right)\right]\right] \leq C_{\bar{x}, \delta} \lambda(1-\lambda)|x-\bar{x}|^{2} .
$$

Similar estimates hold for the term involving $g$ in 101 , and then by 102,103 and 112 we conclude that 89. holds true for $V_{k}^{h}(\cdot)$.

Remark 4.3. If in addition to the above assumptions, we assume that the cost functionals $\ell$ and $g$ are Lipschitz or $f$ and $\sigma$ are affine, then similar arguments as those in the previous proof (see [30, Proposition 4.5, p.187]) show that $V$ and $V_{k}^{h}$ satisfy (89) for some $K$ (independent of $h$ in the case of the discrete value function).

Now we can define for each $h=\frac{T}{N}$, the discrete value function, $V^{h}:[0, T] \times \mathbb{R}^{n} \rightarrow \mathbb{R}$ as a linear interpolation in time of the functions $V_{k}^{h}$, i.e.

$$
V^{h}(t, x):=\alpha V_{k}^{h}(x)+(1-\alpha) V_{k+1}^{h}(x),
$$

for $t=\alpha t_{k}+(1-\alpha) t_{k+1}, \alpha \in[0,1)$. Combining Theorem 4.1 and Theorem 4.2, we easily obtain the following result. 
Theorem 4.4. Under assumptions (H1) and (H2), for each $h=\frac{T}{N}$ the discrete value function $V^{h}$ is $\frac{1}{2}$-Hölder continuous in time. If in addition, we assume that $\mathbf{( H 3 )}$ and $\mathbf{( H 4 )}$ hold, then $V^{h}$ is locally semi-concave in the second variable.

Proof. Let $s, t \in[0, T]$ with $s<t$. There exist $k_{s}, k_{t} \in\{0, \ldots, N-1\}$ such that $s \in\left[t_{k_{s}}, t_{k_{s}+1}\right)$ and $t \in\left[t_{k_{t}}, t_{k_{t}+1}\right)$. Then, there exists $\alpha_{i} \in[0,1)$, such that $i=\alpha_{i} t_{k_{i}}+\left(1-\alpha_{i}\right) t_{k_{i}+1}$, for $i=s, t$.

If $k_{s}=k_{t}$, by the definition of $V^{h}$ we obtain, for all $x \in \mathbb{R}^{n}$,

$$
\begin{aligned}
\left|V^{h}(s, x)-V^{h}(t, x)\right| & \leq\left|\alpha_{s} V_{k_{s}}^{h}(x)+\left(1-\alpha_{s}\right) V_{k_{s}+1}^{h}(x)-\alpha_{t} V_{k_{s}}^{h}(x)-\left(1-\alpha_{t}\right) V_{k_{s}+1}^{h}(x)\right| \\
& \leq\left|\alpha_{s}-\alpha_{t}\right|\left|V_{k_{s}}^{h}(x)-V_{k_{s}+1}^{h}(x)\right| \\
& \leq C\left[1+|x|^{2}\right]\left|\alpha_{s}-\alpha_{t}\right| h^{\frac{1}{2}}
\end{aligned}
$$

where the last inequality holds by Theorem 4.1 Since $|s-t|=\left|\alpha_{s}-\alpha_{t}\right| h$ and $\left|\alpha_{s}-\alpha_{t}\right|<1$, we deduce that

$$
\left|V^{h}(s, x)-V^{h}(t, x)\right| \leq C\left[1+|x|^{2}\right]|s-t|^{\frac{1}{2}} .
$$

Now, assume that $k_{s}<k_{t}$. Notice that

$$
|s-t|=\left[\alpha_{s}+\left(k_{t}-k_{s}-1\right)+\left(1-\alpha_{t}\right)\right] h .
$$

By Theorem 4.1 we have

$$
\begin{aligned}
\left|V^{h}(s, x)-V^{h}(t, x)\right|^{2} \leq & 3\left[\left|\alpha_{s} V_{k_{s}}^{h}(x)+\left(1-\alpha_{s}\right) V_{k_{s}+1}^{h}(x)-V_{k_{s}+1}^{h}(x)\right|^{2}+\left|V_{k_{s}+1}^{h}(x)-V_{k_{t}}^{h}(s)\right|^{2}\right. \\
& \left.+\left|V_{k_{t}}^{h}(x)-\alpha_{t} V_{k_{t}}^{h}(x)+\left(1-\alpha_{t}\right) V_{k_{t}+1}^{h}(x)\right|^{2}\right] \\
\leq & 3\left[\left|\alpha_{s}\right|^{2}\left|V_{k_{s}}^{h}(x)-V_{k_{s}+1}^{h}(x)\right|^{2}+\left|V_{k_{s}+1}^{h}(x)-V_{k_{t}}^{h}(s)\right|^{2}\right. \\
& \left.\quad+\left|1-\alpha_{t}\right|^{2}\left|V_{k_{t}}^{h}(x)-V_{k_{t}+1}^{h}(s)\right|^{2}\right] \\
\leq & 3 C^{2}\left[1+|x|^{2}\right]^{2}\left[\left|\alpha_{s}\right|^{2} h+\left(k_{t}-k_{s}-1\right) h+\left|1-\alpha_{t}\right|^{2} h\right] \\
\leq & 3 C^{2}\left[1+|x|^{2}\right]^{2}\left[\alpha_{s}+\left(k_{t}-k_{s}-1\right)+\left(1-\alpha_{t}\right)\right] h,
\end{aligned}
$$

where the last inequality holds since $\alpha_{s},\left(1-\alpha_{t}\right) \in[0,1]$. Finally, by (116) and 117], for all $x \in \mathbb{R}^{n}$ and $s, t \in[0, T]$ we have

$$
\left|V^{h}(s, x)-V^{h}(t, x)\right| \leq \sqrt{3} C\left[1+|x|^{2}\right]|s-t|^{\frac{1}{2}} .
$$

Since the constant $K_{\bar{x}, \delta}$ in Theorem 4.2 is independent of $k=0, \ldots, N$, for $t=\alpha t_{k}+(1-\alpha) t_{k+1}$, and for all $\lambda \in[0,1]$, we have

$$
\begin{aligned}
\lambda V^{h}(t, x)+(1-\lambda) V^{h}(t, \bar{x})- & V^{h}(t, \lambda x+(1-\lambda) \bar{x}) \\
= & \alpha\left[\lambda V_{k}^{h}(x)+(1-\lambda) V_{k}^{h}(\bar{x})-V_{k}^{h}(\lambda x+(1-\lambda) \bar{x})\right] \\
& +(1-\alpha)\left[\lambda V_{k+1}^{h}(x)+(1-\lambda) V_{k+1}^{h}(\bar{x})-V_{k+1}^{h}(\lambda x+(1-\lambda) \bar{x})\right] \\
\leq & K_{\bar{x}, \delta} \lambda(1-\lambda)|x-\bar{x}|^{2},
\end{aligned}
$$

for all $x \in B_{\delta}(\bar{x})$. The result follows. 


\section{Convergence}

In this section we will analyse the relationship between the value of the discrete and the continuous problems. As the time step $h$ tends to zero we will prove that $V^{h}$ converges to $V$ and also that any sequence of solutions of the discrete problems, extended as piecewise constant processes in $[0, T]$, is a minimizing sequence for the continuous problem. In particular, we can take as minimizing sequence the one consisting on the discrete time feedback controls constructed in Proposition 3.6 and Remark 3.7. Finally, under some convexity assumptions, we can prove the weak convergence of the discrete optimal controls to a solution of the continuous problem.

Throughout this section we will assume that (H1)-(H2) hold. We begin by providing an estimate on the difference between the cost functions of the discrete and the continuous problems. In what follows, for any $k=0, \ldots, N-1$ and $x \in \mathbb{R}^{n}$, we extend $J_{k}^{h}(x, \cdot)$ to $\Pi_{i=0}^{N-1} L_{\mathcal{F}_{t_{i}}^{0}}^{2}$ using the same r.h.s. as in $(9)$.

Lemma 5.1. Let $u^{h} \in \Pi_{i=0}^{N-1} L_{\mathcal{F}_{t_{i}}^{0}}^{2}$ and define the control in $\mathcal{U}_{a d}^{0}$, still denoted by $u^{h}$, as $u^{h}(t):=u_{k}^{h}$ for all $t \in\left[t_{k}, t_{k+1}\right)$. Then, for all $k=0, \ldots, N-1$ there exists $C>0$ independent of $u^{h}$ and $k$ such that

$$
\left|J_{k}^{h}\left(x, u^{h}\right)-J^{t_{k}, x}\left(u^{h}\right)\right| \leq C\left[|x|^{2}+1\right] h^{\frac{1}{2}}+C\left[|x|^{2}+1\right]^{\frac{1}{2}} \bar{\omega}(h)
$$

Proof. For notational convenience we will assume that $k=0$ and, since $x$ is fixed, we denote $J^{h}\left(u^{h}\right)=J_{0}^{h}\left(x, u^{h}\right)$ and $J\left(u^{h}\right)=J^{0, x}\left(u^{h}\right)$. Let $\tilde{y}^{h}$ be the continuous solution of the state equation with respect to the control $u^{h}$, and $y^{h}=\left(y_{k}^{h}\right)_{k=0}^{N}$ the discrete state associated with $u^{h}$. We have

$$
\left|J\left(u^{h}\right)-J^{h}\left(u^{h}\right)\right| \leq\left|\mathbb{E} \sum_{k=0}^{N-1} \int_{t_{k}}^{t_{k+1}}\left[\ell\left(t, \tilde{y}^{h}(t), u_{k}^{h}\right)-\ell\left(t_{k}, y_{k}^{h}, u_{k}^{h}\right)\right] \mathrm{d} t\right|+\left|\mathbb{E}\left[g\left(\tilde{y}^{h}\left(t_{N}\right)\right)-g\left(y_{N}^{h}\right)\right]\right|
$$

and

$$
\begin{aligned}
\left|\mathbb{E} \int_{t_{k}}^{t_{k+1}}\left[\ell\left(t, \tilde{y}^{h}(t), u_{k}^{h}\right)-\ell\left(t_{k}, y_{k}^{h}, u_{k}^{h}\right)\right] \mathrm{d} t\right| \leq & \mathbb{E}\left|\int_{t_{k}}^{t_{k+1}}\left[\ell\left(t, \tilde{y}^{h}(t), u_{k}^{h}\right)-\ell\left(t, y_{k}^{h}, u_{k}^{h}\right)\right] \mathrm{d} t\right| \\
& +\mathbb{E} \int_{t_{k}}^{t_{k+1}}\left|\ell\left(t, y_{k}^{h}, u_{k}^{h}\right)-\ell\left(t_{k}, y_{k}^{h}, u_{k}^{h}\right)\right| \mathrm{d} t .
\end{aligned}
$$

By (H2), for the last term we obtain,

$$
\mathbb{E} \int_{t_{k}}^{t_{k+1}}\left|\ell\left(t, y_{k}^{h}, u_{k}^{h}\right)-\ell\left(t_{k}, y_{k}^{h}, u_{k}^{h}\right)\right| \mathrm{d} t \leq h \bar{\omega}(h)
$$

and for the first one, again by (H2) we have

$$
\begin{aligned}
\mathbb{E} \mid \int_{t_{k}}^{t_{k+1}}\left[\ell \left(t, \tilde{y}^{h}(t),\right.\right. & \left.\left.u_{k}^{h}\right)-\ell\left(t, y_{k}^{h}, u_{k}^{h}\right)\right] \mathrm{d} t \mid \\
& \leq \mathbb{E} \int_{t_{k}}^{t_{k+1}} \int_{0}^{1}\left|\ell_{y}\left(t, \tilde{y}^{h}(t)+s\left(y_{k}^{h}-\tilde{y}^{h}(t)\right), u_{k}^{h}\right)\right|\left|\tilde{y}^{h}(t)-y_{k}^{h}\right| \mathrm{d} s \mathrm{~d} t \\
& \leq \mathbb{E} \int_{t_{k}}^{t_{k+1}} L\left[\left[\left|\tilde{y}^{h}(t)\right|+\left|u_{k}^{h}\right|+1\right]\left|\tilde{y}^{h}(t)-y_{k}^{h}\right|+\left|\tilde{y}^{h}(t)-y_{k}^{h}\right|^{2}\right] \mathrm{d} t .
\end{aligned}
$$

Since $U_{a d}$ is compact, by the Cauchy-Schwarz inequality, 24, Proposition 2.1] and Lemma 2.4. there exists $C_{0}>0$ such that

$$
\begin{aligned}
\int_{t_{k}}^{t_{k+1}} \mathbb{E}\left[\left|\tilde{y}^{h}(t)\right|\left|\tilde{y}^{h}(t)-y_{k}^{h}\right|\right] \mathrm{d} t & \leq \int_{t_{k}}^{t_{k+1}}\left[\mathbb{E}\left[\left|\tilde{y}^{h}(t)\right|^{2}\right]\right]^{\frac{1}{2}}\left[\mathbb{E}\left[\left|\tilde{y}^{h}(t)-y_{k}^{h}\right|^{2}\right]\right]^{\frac{1}{2}} \mathrm{~d} t \\
& \leq C_{0}\left[|x|^{2}+1\right] h^{\frac{3}{2}}+C_{0}\left[|x|^{2}+1\right]^{\frac{1}{2}} h \bar{\omega}(h),
\end{aligned}
$$


and also

$$
\int_{t_{k}}^{t_{k+1}} \mathbb{E}\left[\left|u_{k}^{h}\right|\left|\tilde{y}^{h}(t)-y_{k}^{h}\right|\right] \mathrm{d} t \leq \int_{t_{k}}^{t_{k+1}}\left[\mathbb{E}\left[\left|u_{k}^{h}\right|^{2}\right]\right]^{\frac{1}{2}}\left[\mathbb{E}\left[\left|\tilde{y}^{h}(t)-y_{k}^{h}\right|^{2}\right]\right]^{\frac{1}{2}} \mathrm{~d} t \leq C_{0}\left[|x|^{2}+1\right]^{\frac{1}{2}} h^{\frac{3}{2}}+C_{0} h \bar{\omega}(h) .
$$

By 124)-126 and Lemma 2.4 there exists $C_{1}>0$ such that

$$
\left|\mathbb{E} \int_{t_{k}}^{t_{k+1}}\left[\ell\left(t, \tilde{y}^{h}(t), u_{k}^{h}\right)-\ell\left(t, y_{k}^{h}, u_{k}^{h}\right)\right] \mathrm{d} t\right| \leq C_{1}\left[|x|^{2}+1\right] h^{\frac{3}{2}}+C_{1}\left[|x|^{2}+1\right]^{\frac{1}{2}} h \bar{\omega}(h) .
$$

Arguing as before we can prove that

$$
\mathbb{E}\left[\left|g\left(\tilde{y}^{h}\left(t_{N}\right)\right)-g\left(y_{N}^{h}\right)\right|\right] \leq C_{1}\left[|x|^{2}+1\right] h^{\frac{1}{2}}+C_{1}\left[|x|^{2}+1\right]^{\frac{1}{2}} \bar{\omega}(h) .
$$

Then, we conclude that there exists $C>0$ such that

$$
\left|J\left(u^{h}\right)-J^{h}\left(u^{h}\right)\right| \leq C\left[|x|^{2}+1\right] h^{\frac{1}{2}}+C\left[|x|^{2}+1\right]^{\frac{1}{2}} \bar{\omega}(h) .
$$

Consider a sequence $\left(N_{j}\right)_{j \in \mathbb{N}} \subset \mathbb{N}$ such that $N_{j} \rightarrow \infty$ as $j \rightarrow \infty$ and define $h_{j}=T / N_{j}$ and $t_{k}=k h_{j}$ $\left(k=0, \ldots, N_{j}\right)$. Let $x \in \mathbb{R}^{n}, t \in(0, T]$ and $\left(\varepsilon_{j}\right)_{j \in \mathbb{N}}$ such that $\varepsilon_{j} \geq 0$ and $\lim _{j \rightarrow \infty} \varepsilon_{j}=0$. Let $\left(\bar{u}^{h_{j}}\right)_{j}$ be a sequence of $\varepsilon_{j}$-optimal controls for the discrete problems associated with $V_{k_{j}+1}^{h}(x)$, where $k_{j} \in\left\{0, \ldots, N_{j}-1\right\}$ is such that $t \in\left(t_{k_{j}}, t_{k_{j+1}}\right]$. Let us define

$$
\tilde{u}^{h_{j}}(s)= \begin{cases}\bar{u}_{k_{j}+1,}^{h_{j}}, & s \in\left[t, t_{k_{j}+1}\right), \\ \bar{u}_{m}^{h_{j}}, & s \in\left[t_{m}, t_{m+1}\right), \quad m=k_{j}+1, \cdots, N_{j}-1 .\end{cases}
$$

In the case $t=0$ we define $\tilde{u}^{h_{j}}(s)=\bar{u}_{m}^{h_{j}}$, for all $s \in\left[t_{m}, t_{m+1}\right)$ and $m=0, \cdots, N_{j}-1$, where $\bar{u}^{h_{j}}$ is an $\varepsilon_{j}$-optimal control for the discrete problem associated with $V^{0}(x)$. Note that by definition $\tilde{u}^{h_{j}} \in \mathcal{U}_{\text {ad }}^{t}$. We point out that $\tilde{u}^{h_{j}}$ depends on $t$, but for notational convenience we have omitted this dependence. Now we prove the main result of this section.

Theorem 5.2. Under the above notations we have

$$
V(t, x)=\lim _{j \rightarrow \infty} V^{h_{j}}(t, x), \forall(t, x) \in[0, T] \times \mathbb{R}^{n},
$$

where $V^{h_{j}}$ was defined in 113 and

$$
V(t, x)=\lim _{j \rightarrow \infty} J^{t, x}\left(\tilde{u}^{h_{j}}\right) .
$$

In addition, if $K \subset \mathbb{R}^{n}$ is a compact set, the sequence $\left(V^{h_{j}}\right)_{j}$ converges uniformly to $V$ on $[0, T] \times K$.

Proof. Let us first show the pointwise convergence in 131 . Let $t \in[0, T]$ and $x \in \mathbb{R}^{n}$ be fixed. For each $h_{j}$, we consider the partition of $[0, T]$ given by $\left\{t_{0}, t_{1} \cdots, t_{N_{j}}\right\}$ where $t_{k}=k h_{j}$, for $k=0, \cdots, N_{j}$. Thus, if $t \in(0, T]$ for all $j \in \mathbb{N}$ there exists $k_{j}$ such that $t \in\left(t_{k_{j}}, t_{k_{j}+1}\right]$. If $t=0$, we denote $t_{k_{j}+1}=0$. Let $\varepsilon$ be a positive number, then there exists an $\frac{\varepsilon}{2}$-optimal control $\bar{u}_{\varepsilon} \in \mathcal{U}_{a d}^{t}$ such that

$$
J^{t, x}\left(\bar{u}_{\varepsilon}\right) \leq V(t, x)+\frac{\varepsilon}{2}
$$

For all $j$ large enough we have

$$
J^{t_{k_{j}+1}, x}\left(\bar{u}_{\varepsilon}\right) \leq V\left(t_{k_{j}+1}, x\right)+\varepsilon \text {. }
$$


Indeed, by 133 and Theorem 4.1 there exists $C_{0}>0$, independent of $h_{j}$, such that,

$$
\begin{aligned}
J^{t_{k_{j}+1}, x}\left(\bar{u}_{\varepsilon}\right) & =J^{t_{k_{j}+1}, x}\left(\bar{u}_{\varepsilon}\right)-J^{t, x}\left(\bar{u}_{\varepsilon}\right)+J^{t, x}\left(\bar{u}_{\varepsilon}\right) \\
& \leq C_{0}\left[1+|x|^{2}\right] h_{j}^{\frac{1}{2}}+V(t, x)+\frac{\varepsilon}{2} \\
& \leq C_{0}\left[1+|x|^{2}\right] 2 h_{j}^{\frac{1}{2}}+V\left(t_{k_{j}+1}, x\right)+\frac{\varepsilon}{2} .
\end{aligned}
$$

Let $\left(\varepsilon_{j}\right)_{j \in \mathbb{N}}$ be a decreasing sequence of positive numbers that converges to 0 . Then, there exists an $\varepsilon_{j}$-optimal control, $\bar{u}^{h_{j}} \in \mathcal{U}_{k_{j}+1}^{h_{j}}$ for each $V^{h_{j}}\left(t_{k_{j}+1}, x\right)$, i.e.

$$
V^{h_{j}}\left(t_{k_{j}+1}, x\right) \leq J_{k_{j}+1}^{h_{j}}\left(x, \bar{u}^{h_{j}}\right) \leq V^{h_{j}}\left(t_{k_{j}+1}, x\right)+\varepsilon_{j} .
$$

Since $U_{a d}$ is compact, by a result of Krylov (see 19, Lemma 6, Section 3.2, p.143]), for any $\varepsilon^{\prime}>0$, there exists $N_{\varepsilon^{\prime}}$ such that for all $j \geq N_{\varepsilon^{\prime}}$ there exists $u^{h_{j}} \in \mathbb{H}_{\mathbb{F}^{0}}^{2}$ constant in each interval of the partition $\left\{t, t_{k_{j}+1}, \ldots, t_{N_{j}}\right\}$, such that

$$
\left\|u^{h_{j}}-\bar{u}_{\varepsilon}\right\|_{\mathbb{F}^{2}}<\varepsilon^{\prime} .
$$

It is clear that $u^{h_{j}}=\left(u_{t}^{h_{j}}, u_{k_{j}+1}^{h_{j}}, \cdots, u_{N_{j}}^{h_{j}}\right)$ belongs to $L_{\mathcal{F}_{t}^{0}}^{2} \times \Pi_{i=k_{j}+1}^{N-1} L_{\mathcal{F}_{t_{i}}^{0}}^{2}$. We can define $\hat{u}^{h_{j}}=\left(u_{k_{j}+1}^{h_{j}}, \cdots, u_{N_{j}}^{h_{j}}\right) \in$ $\Pi_{i=k_{j}+1}^{N-1} L_{\mathcal{F}_{t_{i}}^{0}}^{2}$, then, using the same notations that in the previous lemma, we obtain

$$
\begin{aligned}
\left|J^{t, x}\left(\bar{u}_{\varepsilon}\right)-J_{k_{j}+1}^{h_{j}}\left(x, \hat{u}^{h_{j}}\right)\right| \leq & \left|J^{t, x}\left(\bar{u}_{\varepsilon}\right)-J^{t, x}\left(u^{h_{j}}\right)\right|+\left|J^{t, x}\left(u^{h_{j}}\right)-J^{t_{k_{j}+1}, x}\left(\hat{u}^{h_{j}}\right)\right| \\
& +\left|J^{t_{k_{j}+1}, x}\left(\hat{u}^{h_{j}}\right)-J_{k_{j}+1}^{h_{j}}\left(x, \hat{u}^{h_{j}}\right)\right| .
\end{aligned}
$$

By (137) and the continuity of $J^{t, x}$ in $\mathcal{U}_{a d}^{0}$ we deduce the first term in the r.h.s. goes to zero when $j$ goes to infinity. Since $u^{h_{j}}$ and $\hat{u}^{h_{j}}$ coincide from the time $t_{k_{j}+1}$, by Theorem 4.1, there exists $C_{1}>0$ such that,

$$
\left|J^{t, x}\left(u^{h_{j}}\right)-J^{t_{k_{j}+1}, x}\left(\hat{u}^{h_{j}}\right)\right| \leq C_{1}\left[1+|x|^{2}\right] h_{j}^{\frac{1}{2}},
$$

and by Lemma 5.1, there exists $C_{2}>0$ such that,

$$
\left|J^{t_{k_{j}+1}, x}\left(\hat{u}^{h_{j}}\right)-J_{k_{j}+1}^{h_{j}}\left(x, \hat{u}^{h_{j}}\right)\right| \leq C_{2}\left[1+|x|^{2}\right]\left[h_{j}^{\frac{1}{2}}+\bar{\omega}\left(h_{j}\right)\right] .
$$

Therefore, we can conclude that for $j$ large enough we have,

$$
\left|J^{t, x}\left(\bar{u}_{\varepsilon}\right)-J_{k_{j}+1}^{h_{j}}\left(x, \hat{u}^{h_{j}}\right)\right|<\varepsilon .
$$

As we saw in Remark 3.5, the value $V^{h_{j}}\left(t_{k_{j}+1}, x\right)$ is the same as if we minimize over the set of controls $\Pi_{i=k_{j}+1}^{N_{j}-1} L_{\mathcal{F}_{t_{i}}^{0}}^{2}$ and, since $\hat{u}^{h_{j}}$ belongs to this set, we have

$$
V^{h_{j}}\left(t_{k_{j}+1}, x\right) \leq J_{k_{j}+1}^{h_{j}}\left(x, \hat{u}^{h_{j}}\right) \leq J^{t, x}\left(\bar{u}_{\varepsilon}\right)+\varepsilon \leq V(t, x)+2 \varepsilon,
$$

where in the last two inequalities we have used $(141)$ and $(133)$. On the other hand, Theorem 4.1 implies that there exists $C_{3}>0$ such that

$$
V(t, x) \leq C_{3}\left[1+|x|^{2}\right] h_{j}^{\frac{1}{2}}+V\left(t_{k_{j}+1}, x\right) .
$$


Finally, Lemma 5.1 and 136 yield the existence of $C_{4}>0$ such that

$$
\begin{aligned}
V\left(t_{k_{j}+1}, x\right) & \leq J^{t_{k_{j}+1}, x}\left(\bar{u}^{h_{j}}\right) \leq J_{k_{j}+1}^{h_{j}}\left(x, \bar{u}^{h_{j}}\right)+C_{4}\left[1+|x|^{2}\right]\left[h_{j}^{\frac{1}{2}}+\bar{\omega}\left(h_{j}\right)\right] \\
& \leq V^{h_{j}}\left(t_{k_{j}+1}, x\right)+\varepsilon_{j}+C_{4}\left[1+|x|^{2}\right]\left[h_{j}^{\frac{1}{2}}+\bar{\omega}\left(h_{j}\right)\right] .
\end{aligned}
$$

Combining the last three inequalities and using Theorem 4.4, we get the existence of $C>0$ such that for $j$ large enough,

$$
\begin{aligned}
\left|V(t, x)-V^{h_{j}}(t, x)\right| & \leq\left|V(t, x)-V^{h_{j}}\left(t_{k_{j}+1}, x\right)\right|+\left|V^{h_{j}}\left(t_{k_{j}+1}, x\right)-V^{h_{j}}(t, x)\right| \\
& \leq \varepsilon_{j}+2 \varepsilon+C\left[\left[1+|x|^{2}\right]\left[h_{j}^{\frac{1}{2}}+\bar{\omega}\left(h_{j}\right)\right] .\right.
\end{aligned}
$$

Letting $j \uparrow \infty$ and using that $\varepsilon>0$ is arbitrary we obtain (131).

Now, let us prove (132). Combining Theorem 4.1 and Lemma 5.1, we get the existence of $C>0$ such that

$$
\begin{aligned}
\left|J^{t, x}\left(\tilde{u}^{h_{j}}\right)-J_{k_{j}+1}^{h_{j}}\left(x, \bar{u}^{h_{j}}\right)\right| & \leq\left|J^{t, x}\left(\tilde{u}^{h_{j}}\right)-J^{t_{k_{j}+1}, x}\left(\tilde{u}^{h_{j}}\right)\right|+\left|J^{t_{k_{j}+1}, x}\left(\bar{u}^{h_{j}}\right)-J_{k_{j}+1}^{h_{j}}\left(x, \bar{u}^{h_{j}}\right)\right| \\
& \leq C\left[1+|x|^{2}\right]\left[h_{j}^{\frac{1}{2}}+\bar{\omega}\left(h_{j}\right)\right] .
\end{aligned}
$$

By (144) and 131, we conclude

$$
\lim _{j \rightarrow \infty} J^{t, x}\left(\tilde{u}^{h_{j}}\right)=\lim _{j \rightarrow \infty} J_{k_{j}+1}^{h_{j}}\left(x, \bar{u}^{h_{j}}\right)=V(t, x)
$$

In order to prove the last assertion of the theorem, let $K \subset \mathbb{R}^{n}$ be a compact set. By Theorem4.1 and Lemma 3.1 we deduce that the sequence $\left(V^{h_{j}}\right)_{j}$ is uniformly bounded and uniformly equicontinuous on $[0, T] \times K$. Then, by the Ascoli-Arzelá theorem, and the pointwise convergence (131), we deduce that the sequence uniformly converges to $V$ on $[0, T] \times K$.

Remark 5.3. We emphasize that our direct approach allows us to deal with more general assumptions that the usually considered in the literature, such as, coefficients which are bounded and/or independence of the time variable. Also an important consequence of relation 132 is that $\left(\tilde{u}^{h_{j}}\right)$ is a minimizing sequence for the optimal control problem associated with $V(t, x)$. In particular, we can take as $\left(\tilde{u}^{h_{j}}\right)$ the sequence of discrete time feedback controls constructed in Remark 3.7

The following result shows that under some convexity assumptions, we have convergence of $\left(\tilde{u}^{h_{j}}\right)$ to an optimal solution of the continuous problem.

Corollary 5.4. Suppose in addition that $U_{a d}$ is a convex set and $J^{t, x}$ is a convex functional. Then, there exists at least one weak limit point of $\left(\tilde{u}^{h_{j}}\right)$, and any limit point $u^{*} \in \mathcal{U}_{\text {ad }}^{t}$ satisfies,

$$
J^{t, x}\left(u^{*}\right)=V(t, x)
$$

If in addition, $J^{t, x}$ is strongly convex, then the whole sequence $\left(\tilde{u}^{h_{j}}\right)_{j \in \mathbb{N}}$ strongly converges to the unique $u \in \mathcal{U}_{\text {ad }}^{t}$ that verifies 148 .

Proof. First, note that since $U_{a d}$ is compact, the space $\mathcal{U}_{a d}^{t}$ is bounded in $\mathbb{H}_{\mathbb{F}^{t}}^{2}$. Using that the convexity and continuity of $J^{t, x}$ imply its weak lower semi-continuity, classical arguments yield the existence of at least one optimal control $\bar{u}$ for $V(t, x)$.

Since $\tilde{u}^{h_{j}}$ is a bounded sequence in $\mathbb{H}_{\mathbb{F}^{t}}^{2}$, there exists a subsequence (still denoted $\tilde{u}^{h_{j}}$ ) which converges weakly to $u^{*} \in \mathbb{H}_{\mathbb{F}^{t}}^{2}$. By the weak lower semi-continuity and equation 132 we have

$$
J^{t, x}\left(u^{*}\right) \leq \liminf _{j \rightarrow \infty} J^{t, x}\left(\tilde{u}^{h_{j}}\right)=V(t, x) .
$$


Finally, if $J^{t, x}$ is strongly convex, the strong convergence follows from the classical argument stating that a minimizing sequence of a strongly convex problem converge strongly to the unique optimizer of the problem (see e.g. 4, Proof of Lemma 2.33(ii)]).

Remark 5.5. It is worth mentioning that the assumption of convexity holds, for instance, when for a.a. $t \in[0, T]$, the maps $(y, u) \mapsto \ell(t, y, u)$ and $y \mapsto g(y)$ are convex and for $\psi=f, \sigma$ the map $(y, u) \mapsto \psi(t, y, u)$ is affine. If in addition, for some $\varepsilon>0,(y, u) \mapsto \ell(t, y, u)-\varepsilon|u|^{2}$ is convex for a.a. $t \in[0, T]$, then $J^{t, x}$ is a strongly convex function.

\section{APPENDIX}

Here we prove some technical results stated in Subsection 2.4.

Proof of Lemma 2.2, For all $k=1, \ldots, N$ we have

$$
y_{k}=x+h \sum_{j=0}^{k-1} f\left(t_{j}, y_{j}, u_{j}\right)+\sum_{j=0}^{k-1} \sigma\left(t_{j}, y_{j}, u_{j}\right) \Delta W_{j+1} .
$$

Then, by (H1)-(b) there exists $C_{0}>0$ such that,

$$
\begin{aligned}
\left|y_{k}\right|^{2} & \leq 3\left[|x|^{2}+N h^{2} \sum_{j=0}^{k-1}\left|f\left(t_{j}, y_{j}, u_{j}\right)\right|^{2}+\left(\sum_{j=0}^{k-1} \sigma\left(t_{j}, y_{j}, u_{j}\right) \Delta W_{j+1}\right)^{2}\right] \\
& \leq C_{0}\left[|x|^{2}+h \sum_{j=0}^{k-1}\left[\left|y_{j}\right|^{2}+\left|u_{j}\right|^{2}+1\right]+\left(\sum_{j=0}^{k-1} \sigma\left(t_{j}, y_{j}, u_{j}\right) \Delta W_{j+1}\right)^{2}\right] .
\end{aligned}
$$

By the Doob's maximal inequality (see [16, Chapter 2, Theorem 6.10]), the Itô isometry, and (H1)-(b), there exist $C_{1}>0$ and $C_{2}>0$ such that

$$
\begin{aligned}
\mathbb{E}\left[\max _{0 \leq i \leq k}\left|y_{i}\right|^{2}\right] \leq & C_{0}\left[|x|^{2}+h \sum_{j=0}^{k-1}\left[\mathbb{E}\left[\max _{0 \leq i \leq j}\left|y_{i}\right|^{2}\right]+\mathbb{E}\left[\left|u_{j}\right|^{2}\right]+1\right]\right. \\
& \left.+C_{1} \mathbb{E}\left[h \sum_{j=0}^{k-1}\left|\sigma\left(t_{j}, y_{j}, u_{j}\right)\right|^{2}\right]\right] \\
\leq & C_{2}\left[|x|^{2}+h \sum_{j=0}^{k-1}\left[\mathbb{E}\left[\max _{0 \leq i \leq j}\left|y_{i}\right|^{2}\right]+\mathbb{E}\left[\left|u_{j}\right|^{2}\right]+1\right]\right] .
\end{aligned}
$$

The results follows by the discrete Grönwall's lemma (see, e.g. [10]).

Proof of Lemma 2.3. Denoting $\Delta y_{j}=y_{j}^{x}-y_{j}^{y}$, and $\Delta \varphi_{j}=\varphi\left(t_{j}, y_{j}^{x}, u_{j}\right)-\varphi\left(t_{j}, y_{j}^{y}, u_{j}\right)$ for $\varphi=f, \sigma$, we obtain for $i=0, \ldots N-1$,

$$
\Delta y_{i+1}=x-y+\sum_{j=0}^{i} h \Delta f_{j}+\sum_{j=0}^{i} \Delta \sigma_{j} \Delta W_{j+1}
$$

We have,

$$
\left|\Delta y_{i+1}\right|^{p} \leq 3^{p-1}\left[|x-y|^{p}+\left|\sum_{j=0}^{i} h \Delta f_{j}\right|^{p}+\left|\sum_{j=0}^{i} \Delta \sigma_{j} \Delta W_{j+1}\right|^{p}\right] .
$$

By (H1) we get

$$
\left|\sum_{j=0}^{i} h \Delta f_{j}\right|^{p} \leq N^{p-1} h^{p} \sum_{j=0}^{i}\left|\Delta f_{j}\right|^{p} \leq T^{p-1} h L^{p} \sum_{j=0}^{i}\left|\Delta y_{j}\right|^{p}
$$


Now, by (H1) and the Burkholder-Davis-Gundy inequality [6], there exists $K_{p}$ (independent of $h$ ), such that

$$
\begin{aligned}
\mathbb{E}\left(\max _{0 \leq m \leq i}\left|\sum_{j=0}^{m} \Delta \sigma_{j} \Delta W_{j+1}\right|^{p}\right) & \leq K_{p} \mathbb{E}\left(\left[h \sum_{j=0}^{i}\left|\Delta \sigma_{j}\right|^{2}\right]^{\frac{p}{2}}\right) \\
& \leq K_{p} N^{\frac{p}{2}-1} h^{\frac{p}{2}} \sum_{j=0}^{i} \mathbb{E}\left(\left|\Delta \sigma_{j}\right|^{p}\right) \\
& \leq K_{p} T^{\frac{p}{2}-1} h L^{p} \sum_{j=0}^{i} \mathbb{E}\left(\left|\Delta y_{j}\right|^{p}\right) .
\end{aligned}
$$

Combining (154, 155) and 156), there exists $c_{p}$ such that

$$
\mathbb{E}\left(\max _{0 \leq m \leq i+1}\left|\Delta y_{m}\right|^{p}\right) \leq c_{p}|x-y|^{p}+c_{p} h \sum_{j=0}^{i} \mathbb{E}\left(\max _{0 \leq m \leq j}\left|\Delta y_{m}\right|^{p}\right) .
$$

The conclusion follows from the discrete Grönwall's lemma.

Finally, for the last result of Subsection 2.4, we need the following lemma. We recall that $y(\cdot)$ is the solution of (20) and $\left(y_{k}\right)_{k=0}^{N}$ is the solution of (8) associated with $u_{c}^{h}$ and $u^{h}$, respectively, where for notational convenience we have omitted the indexes of the initial time and the initial condition.

Lemma A.1. Assume that (H1) holds true. Then, there exists $C>0$ such that

$$
\max _{k=0, \ldots, N} \mathbb{E}\left|y\left(t_{k}\right)-y_{k}\right|^{2} \leq C h\left[|x|^{2}+\left\|u^{h}\right\|_{\mathcal{U}_{0}^{h}}^{2}+1\right]+C \bar{\omega}^{2}(h),
$$

for all $k=0, \ldots, N$.

Proof. For all $k=0, \ldots, N-1$ we define $\Delta y_{k}:=y\left(t_{k}\right)-y_{k}$,

$$
\Delta f_{k}(t):=f\left(t, y(t), u_{k}\right)-f\left(t_{k}, y_{k}, u_{k}\right), \quad \text { and } \quad \Delta \sigma_{k}(t):=\sigma\left(t, y(t), u_{k}\right)-\sigma\left(t_{k}, y_{k}, u_{k}\right)
$$

We have,

$$
\Delta y_{k+1}=\Delta y_{k}+\int_{t_{k}}^{t_{k+1}} \Delta f_{k}(t) \mathrm{d} t+\int_{t_{k}}^{t_{k+1}} \Delta \sigma_{k}(t) \mathrm{d} W(t) .
$$

Therefore, by the Cauchy-Schwarz inequality and taking conditional expectation inside the expectation, we obtain

$$
\begin{aligned}
\mathbb{E}\left[\left|\Delta y_{k+1}\right|^{2}\right] \leq & \mathbb{E}\left[\left|\Delta y_{k}\right|^{2}\right]+\mathbb{E}\left[\left|\int_{t_{k}}^{t_{k+1}} \Delta f_{k}(t) \mathrm{d} t\right|^{2}\right]+\mathbb{E}\left[\left|\int_{t_{k}}^{t_{k+1}} \Delta \sigma_{k}(t) \mathrm{d} W(t)\right|^{2}\right] \\
& +2\left(\mathbb{E}\left[\left|\Delta y_{k}\right|^{2}\right]\right)^{\frac{1}{2}}\left(\mathbb{E}\left[\left|\int_{t_{k}}^{t_{k+1}} \Delta f_{k}(t) \mathrm{d} t\right|^{2}\right]\right)^{\frac{1}{2}} \\
& +2\left(\mathbb{E}\left[\left|\int_{t_{k}}^{t_{k+1}} \Delta f_{k}(t) \mathrm{d} t\right|^{2}\right]\right)^{\frac{1}{2}}\left(\mathbb{E}\left[\left|\int_{t_{k}}^{t_{k+1}} \Delta \sigma_{k}(t) \mathrm{d} W(t)\right|^{2}\right]\right)^{\frac{1}{2}} .
\end{aligned}
$$

Applying the Young's inequality in the last two terms, we have

$$
\begin{gathered}
\mathbb{E}\left[\left|\Delta y_{k+1}\right|^{2}\right] \leq \quad[1+h] \mathbb{E}\left[\left|\Delta y_{k}\right|^{2}\right]+\left[1+\frac{2}{h}\right] \mathbb{E}\left[\left|\int_{t_{k}}^{t_{k+1}} \Delta f_{k}(t) \mathrm{d} t\right|^{2}\right] \\
+[1+h] \mathbb{E}\left[\left|\int_{t_{k}}^{t_{k+1}} \Delta \sigma_{k}(t) \mathrm{d} W(t)\right|^{2}\right] .
\end{gathered}
$$


Now, we study all the square terms in the r.h.s. of 162 separately. By the Jensen's inequality, we obtain

$$
\begin{aligned}
\mathbb{E}\left[\left|\int_{t_{k}}^{t_{k+1}} \Delta f_{k}(t) \mathrm{d} t\right|^{2}\right] \leq & h \mathbb{E}\left[\int_{t_{k}}^{t_{k+1}}\left|\Delta f_{k}(t)\right|^{2} \mathrm{~d} t\right] \\
\leq & 2 h \mathbb{E} \int_{t_{k}}^{t_{k+1}}\left|f\left(t, y(t), u_{k}\right)-f\left(t, y_{k}, u_{k}\right)\right|^{2} \mathrm{~d} t \\
& +2 h \mathbb{E} \int_{t_{k}}^{t_{k+1}}\left|f\left(t, y_{k}, u_{k}\right)-f\left(t_{k}, y_{k}, u_{k}\right)\right|^{2} \mathrm{~d} t
\end{aligned}
$$

and by (H1), we get

$$
\begin{aligned}
\mathbb{E}\left[\left|\int_{t_{k}}^{t_{k+1}} \Delta f_{k}(t) \mathrm{d} t\right|^{2}\right] & \leq 2 h\left[\int_{t_{k}}^{t_{k+1}} L^{2} \mathbb{E}\left|y(t)-y_{k}\right|^{2} \mathrm{~d} t+\int_{t_{k}}^{t_{k+1}} \bar{\omega}^{2}\left(\left|t-t_{k}\right|\right) \mathrm{d} t\right] \\
& \leq 2 h L^{2} \int_{t_{k}}^{t_{k+1}} \mathbb{E}\left|y(t)-y_{k}\right|^{2} \mathrm{~d} t+2 h^{2} \bar{\omega}^{2}(h) .
\end{aligned}
$$

In order to estimate the integral term in 164, note that for all $t_{k} \leq t<t_{k+1}$ we have,

$$
y(t)-y_{k}=\Delta y_{k}+\int_{t_{k}}^{t} f(s, y(s), u(s)) \mathrm{d} s+\int_{t_{k}}^{t} \sigma(s, y(s), u(s)) \mathrm{d} W(s) .
$$

Then, by (H1), the Cauchy-Schwarz inequality and the Itô isometry, we deduce that there exist $c_{0}>0$ and $C_{0}>0$ such that for all $t_{k} \leq t<t_{k+1}$,

$$
\begin{aligned}
\mathbb{E}\left[\left|y(t)-y_{k}\right|^{2}\right] \leq & c_{0} \mathbb{E}\left[\left|\Delta y_{k}\right|^{2}\right]+c_{0} h \mathbb{E}\left[\int_{t_{k}}^{t_{k+1}}\left[|y(t)|^{2}+\left|u_{k}\right|^{2}+1\right] \mathrm{d} t\right] \\
& +c_{0} \mathbb{E}\left[\int_{t_{k}}^{t_{k+1}}\left[|y(t)|^{2}+\left|u_{k}\right|^{2}+1\right] \mathrm{d} t\right] \\
\leq & C_{0} \mathbb{E}\left[\left|\Delta y_{k}\right|^{2}\right]+C_{0} h\left[|x|^{2}+\left\|u^{h}\right\|_{\mathcal{U}_{0}^{h}}^{2}+\mathbb{E}\left|u_{k}\right|^{2}+1\right]
\end{aligned}
$$

where the last inequality follows from 24, Proposition 2.1]. So, by 164 and (166), there exist positive constants $C_{1}$ and $C_{2}$ such that,

$$
\mathbb{E}\left[\left|\int_{t_{k}}^{t_{k+1}} \Delta f_{k}(t) \mathrm{d} t\right|^{2}\right] \leq C_{1} h^{2} \mathbb{E}\left[\left|\Delta y_{k}\right|^{2}\right]+C_{2} h^{3}\left[|x|^{2}+\left\|u^{h}\right\|_{\mathcal{U}_{0}^{h}}^{2}+\mathbb{E}\left|u_{k}\right|^{2}+1\right]+2 h^{2} \bar{\omega}^{2}(h) .
$$

By the Itô isometry and 166 we have,

$$
\begin{aligned}
\mathbb{E}\left[\int_{t_{k}}^{t_{k+1}} \Delta \sigma_{k}(t) \mathrm{d} W(t)\right]^{2} & =\mathbb{E}\left[\int_{t_{k}}^{t_{k+1}}\left|\Delta \sigma_{k}(t)\right|^{2} \mathrm{~d} t\right] \\
& \leq 2 \mathbb{E}\left[\int_{t_{k}}^{t_{k+1}}\left(L^{2}\left|y(t)-y_{k}\right|^{2}+\bar{\omega}^{2}\left(\left|t-t_{k}\right|\right)\right) \mathrm{d} t\right] \\
& \leq C_{3} h \mathbb{E}\left[\left|\Delta y_{k}\right|^{2}\right]+C_{4} h^{2}\left[|x|^{2}+\left\|u^{h}\right\|_{\mathcal{U}_{0}^{h}}^{2}+\mathbb{E}\left|u_{k}\right|^{2}+1\right]+2 h \bar{\omega}^{2}(h),
\end{aligned}
$$

for suitable positive constants $C_{3}$ and $C_{4}$.

Combining (162), 167) and (168) we conclude that there exist $C_{5}>0, C_{6}>0$ and $C_{7}>0$ such that

$$
\mathbb{E}\left[\left|\Delta y_{k+1}\right|^{2}\right] \leq\left[1+C_{5} h\right] \mathbb{E}\left[\left|\Delta y_{k}\right|^{2}\right]+C_{6} h^{2}\left[|x|^{2}+\left\|u^{h}\right\|_{\mathcal{U}_{0}^{h}}^{2}+\mathbb{E}\left|u_{k}\right|^{2}+1\right]+C_{7} h \bar{\omega}^{2}(h) .
$$


Therefore, we deduce that

$$
\begin{aligned}
\mathbb{E}\left[\left|\Delta y_{k+1}\right|^{2}\right] \leq & {\left[1+C_{5} h\right]^{k} \mathbb{E}\left[\left|\Delta y_{0}\right|^{2}\right]+\sum_{j=0}^{k-1}\left[1+C_{5} h\right]^{j} C_{6} h^{2}\left[|x|^{2}+\left\|u^{h}\right\|_{\mathcal{U}_{0}^{h}}^{2}+\mathbb{E}\left|u_{k}\right|^{2}+1\right] } \\
& +\sum_{j=0}^{k-1}\left[1+C_{5} h\right]^{j} C_{7} h \bar{\omega}^{2}(h) \\
\leq & e^{C_{5} T} C_{6} h\left[T|x|^{2}+2\|u\|_{\mathcal{U}_{0}^{h}}^{2}+T\right]+e^{C_{5} T} C_{7} T \bar{\omega}^{2}(h) \\
\leq & C h\left[|x|^{2}+\left\|u^{h}\right\|_{\mathcal{U}_{0}^{h}}^{2}+1\right]+C \bar{\omega}^{2}(h),
\end{aligned}
$$

for a suitable constant $C>0$.

Proof of Lemma 2.4. By (165), the Doob's maximal inequality and the Itô isometry, there exists $K>0$ such that

$$
\begin{aligned}
\mathbb{E}\left[\sup _{t_{k} \leq t<t_{k+1}}\left|y(t)-y_{k}\right|^{2}\right] \leq & 2 \mathbb{E}\left[\left|y\left(t_{k}\right)-y_{k}\right|^{2}\right]+4 h \mathbb{E}\left[\int_{t_{k}}^{t_{k+1}}\left|f\left(s, y(s), u_{k}\right)\right|^{2} \mathrm{~d} s\right] \\
& +4 K \mathbb{E}\left[\int_{t_{k}}^{t_{k+1}}\left|\sigma\left(s, y(s), u_{k}\right)\right|^{2} \mathrm{~d} s\right] .
\end{aligned}
$$

Since $U_{a d}$ is compact, by (H1), Remark 2.1 and the previous lemma we obtain the result.

\section{REFERENCES}

[1] C. Aliprantis and K. Border. Infinite dimensional analysis. A hitchhiker's guide. Springer, Berlin, third edition, 2006.

[2] G. Barles and P. Souganidis. Convergence of approximation schemes for fully nonlinear second order equations. Asymptotic Analysis, 4:271-283, 1991.

[3] D. P. Bertsekas and S. E. Shreve. Stochastic optimal control : the discrete time case. Academic Press New York, 1978.

[4] J. F. Bonnans and A. Shapiro. Perturbation analysis of optimization problems. Springer Series in Operations Research. Springer-Verlag, New York, 2000.

[5] B. Bouchard and N. Touzi. Weak dynamic programming principle for viscosity solutions. SIAM Journal on Control and Optimization, 49(3):948-962, 2011.

[6] D. L. Burkholder, B. J. Davis, and R. F. Gundy. Integral inequalities for convex functions of operators on martingales. In Proceedings of the Sixth Berkeley Symposium on Mathematical Statistics and Probability, Volume 2: Probability Theory, pages 223-240, Berkeley, Calif., 1972. University of California Press.

[7] I. Capuzzo Dolcetta. On a discrete approximation of the Hamilton-Jacobi equation of dynamic programming. Appl. Math. Optim., 10(4):367-377, 1983.

[8] I. Capuzzo-Dolcetta and H. Ishii. Approximate solutions of the Bellman equation of deterministic control theory. Appl. Math. Optim., 11(2):161-181, 1984.

[9] N. Christopeit. Discrete approximation of continuous time stochastic control systems. SIAM Journal on Control and Optimization, 21(1):17-40, 1983.

[10] D. S. Clark. Short proof of a discrete Gronwall inequality. Discrete Applied Mathematics, 16(3):279 - $281,1987$.

[11] K. Debrabant and E. R. Jakobsen. Semi-lagrangian schemes for linear and fully non-linear diffusion equations. Mathematics of Computation, 82(283):1433-1462, 2013.

[12] E. B. Dynkin and A. A. Yushkevich. Controlled Markov processes, volume 235 of Grundlehren der Mathematischen Wissenschaften [Fundamental Principles of Mathematical Sciences]. Springer-Verlag, Berlin-New York, 1979. Translated from the Russian original by J. M. Danskin and C. Holland.

[13] W. H. Fleming and R. W. Rishel. Deterministic and stochastic optimal control. Springer-Verlag, Berlin-New York, 1975. Applications of Mathematics, No. 1.

[14] W. H. Fleming and H. M. Soner. Controlled Markov processes and viscosity solutions, volume 25 of Stochastic Modelling and Applied Probability. Springer, New York, second edition, 2006.

[15] I. I. Gikhman and A. V. Skorohod. Controlled stochastic processes. Springer-Verlag, New York-Heidelberg, 1979. Translated from the Russian by Samuel Kotz.

[16] N. Ikeda and S. Watanabe. Stochastic Differential Equations and Diffusion Processes. North Holland-Kodansha Publishing, 1981.

[17] N. Krylov. Approximating value functions for controlled degenerate diffusion processes by using piece-wise constant policies. Electron. J. Probab., 4:1-19, 1999.

[18] N. V. Krylov. Mean value theorems for stochastic integrals. Ann. Probab., 29(1):385-410, 2001.

[19] N. V. Krylov. Controlled diffusion processes, volume 14. Springer Science \& Business Media, 2008. 
[20] H. Kushner. Probability methods for approximations in stochastic control and for elliptic equations. Academic Press, New York, 1977. Mathematics in Science and Engineering, Vol. 129.

[21] P.-L. Lions. Optimal control of diffusion processes and Hamilton-Jacobi-Bellman equations. I. The dynamic programming principle and applications. Comm. Partial Differential Equations, 8(10):1101-1174, 1983.

[22] P.-L. Lions. Optimal control of diffusion processes and Hamilton-Jacobi-Bellman equations. II. Viscosity solutions and uniqueness. Comm. Partial Differential Equations, 8(11):1229-1276, 1983

[23] P.-L. Lions. Optimal control of diffusion processes and Hamilton-Jacobi-Bellman equations. III. Regularity of the optimal cost function. In Nonlinear partial differential equations and their applications. Collège de France seminar, Vol. V (Paris, 1981/1982), volume 93 of Res. Notes in Math., pages 95-205. Pitman, Boston, MA, 1983.

[24] L. Mou and J. Yong. A variational formula for stochastic controls and some applications. Pure Appl. Math. Q., 3(2, Special Issue: In honor of Leon Simon. Part 1):539-567, 2007.

[25] M. Nisio. Stochastic control theory. Dynamic programming principle. 2nd ed. Tokyo: Springer, 2nd ed. edition, 2015.

[26] L. Pontryagin, V. Boltyanskiı̌, R. Gamkrelidze, and E. Mishchenko. The mathematical theory of optimal processes. Gordon \& Breach Science Publishers, New York, 1986. Reprint of the 1962 English translation.

[27] M. L. Puterman. Markov decision processes: discrete stochastic dynamic programming. Wiley Series in Probability and Mathematical Statistics: Applied Probability and Statistics. John Wiley \& Sons, Inc., New York, 1994. A Wiley-Interscience Publication.

[28] S. Srivastava. A Course on Borel Sets. Springer-Verlag New York, 1998.

[29] N. Touzi. Optimal stochastic control, stochastic target problems, and backward SDE, volume 29 of Fields Institute Monographs. Springer, New York; Fields Institute for Research in Mathematical Sciences, Toronto, ON, 2013. With Chapter 13 by Angès Tourin.

[30] J. Yong and X. Zhou. Stochastic controls, Hamiltonian systems and HJB equations. Springer-Verlag, New York, Berlin, 2000.

[31] A. A. Yushkevich and R. Y. Chitashvili. Controlled random sequences and Markov chains. Russian Mathematical Surveys, $37(6): 239,1982$ 